\title{
Representation of Simple Symmetric Operators with Deficiency Indices $(1,1)$ in de Branges Space
}

\author{
R. T. W. Martin
}

Received: 23 July 2009 / Accepted: 8 September 2009 / Published online: 30 September 2009

(C) The Author(s) 2009. This article is published with open access at Springerlink.com

\begin{abstract}
Recently it has been shown that any regular simple symmetric operator with deficiency indices $(1,1)$ is unitarily equivalent to the operator of multiplication in a reproducing kernel Hilbert space of functions on the real line with the Kramer sampling property. This work has been motivated, in part, by potential applications to signal processing and mathematical physics. In this paper we exploit well-known results about de Branges-Rovnyak spaces and characteristic functions of symmetric operators to prove that any such a symmetric operator is in fact unitarily equivalent to multiplication by the independent variable in a de Branges space of entire functions. This leads to simple new results on the spectra of such symmetric operators, on when multiplication by $z$ is densely defined in de Branges-Rovnyak spaces in the upper half plane, and to sufficient conditions for there to be an isometry from a given subspace of $L^{2}(\mathbb{R}, d v)$ onto a de Branges space of entire functions which acts as multiplication by a measurable function.
\end{abstract}

Keywords Symmetric (isometric) operators with deficiency indices $(1,1)$. Self-adjoint (unitary) extensions · de Branges spaces · de Branges-Rovnyak spaces · Lifschitz characteristic function - Carathéodory or angular derivative

Mathematics Subject Classification (2000) 47B25 (symmetric and self-adjoint operators (unbounded)) · 46E22 (Hilbert spaces with reproducing kernels) · 47B32 (operators in reproducing kernel Hilbert spaces)

Communicated by Aad Dijksma.

R. T. W. Martin ( $\varangle)$

Department of Mathematics, University of California,

Berkeley, Berkeley, CA 94720, USA

e-mail: rtwmartin@gmail.com 


\section{Introduction and Motivation}

A reproducing kernel Hilbert space $\mathcal{H}$ of functions on a set $X \subset \mathbb{C}$ is said to have the Kramer sampling property if it has a total orthogonal set of point evaluation vectors [1]. The reason for this terminology is clear: If $\delta_{x}$ denotes the point evaluation vector in $\mathcal{H}$ at the point $x \in X$, i.e. $\left\langle f, \delta_{x}\right\rangle=f(x)$ for all $f \in \mathcal{H}$, and if $\left\{\delta_{x_{n}}\right\}$ is a total orthogonal set, then any $f \in \mathcal{H}$ can be reconstructed from its 'samples', or values taken on the set of points $\left\{x_{n}\right\} \subset X$, using the sampling formula

$$
f=\sum_{n} f\left(x_{n}\right) \frac{\delta_{x_{n}}}{\delta_{x_{n}}\left(x_{n}\right)} .
$$

We will only consider the case where $\mathcal{H}$ is separable, so that all total orthogonal sets in $\mathcal{H}$ are countable.

The classic examples of such spaces are the Paley-Wiener spaces $B(\Omega)$ of $\Omega$-bandlimited functions, $\Omega>0$. The space $B(\Omega)$ is the image of $L^{2}[-\Omega, \Omega]$ under the Fourier transform, and for $f \in B(\Omega)$ the reconstruction formula (1.1) takes the form

$$
f(x)=\sum_{n \in \mathbb{Z}} f\left(x_{n}\right) \frac{\sin \left(\Omega\left(x-x_{n}\right)\right)}{\Omega\left(x-x_{n}\right)},
$$

where $\left(x_{n}\right) \subset \mathbb{R}$ is any sequence of points satisfying $x_{n+1}-x_{n}=\frac{\pi}{\Omega}$. In this particular case, the formula (1.2) is called the Shannon sampling formula. These spaces are used ubiquitously in signal processing to discretize and later reconstruct continuous signals; e.g. audio signals to be recorded on compact disc, images for digital transmission, etc. The kernel of the idea is that by choosing a sufficiently large bandlimit, a given continuous signal can be well-approximated by a bandlimited function. The samples of this bandlimited approximation on a sufficiently dense set of points can then be recorded and later used in Shannon's sampling formula to reconstruct the approximating function.

There has been a significant amount of recent work focusing on the search for and study of other reproducing kernel Hilbert spaces of functions on the real line with the sampling property (or with the more general property that they contain a Riesz basis or frame of point evaluation vectors) in an effort to develop more efficient methods of discretizing and reconstructing certain classes of continuous signals in signal processing. See, for example [2-7], to name a few. Recently, it has been discovered that there is a connection between the study of such spaces and the spectral representations of symmetric operators with deficiency indices $(1,1)$. In particular, it has been shown that any regular simple symmetric operator, $B$, with deficiency indices $(1,1)$ is unitarily equivalent to multiplication by the independent variable in a reproducing kernel Hilbert space $\mathcal{H}$ with the sampling property where $\mathcal{H} \subset L^{2}(\mathbb{R}, d \sigma)$ has the following properties [4-6,8]. First, the positive measure $\sigma$ can be chosen to be equivalent to Lebesgue measure, in fact $\sigma$ can be chosen such that $d \sigma(x)=\sigma^{\prime}(x) d x$, where $\sigma^{\prime}>0$ is continuous on $\mathbb{R}$. The space $\mathcal{H}$ consists of certain functions which are meromorphic in $\mathbb{C}$ with no poles on the real axis. 
Furthermore, given any self-adjoint extension $B^{\prime}$ of $B$, its spectrum $\sigma\left(B^{\prime}\right)$ is purely discrete and can be arranged as a monotonically strictly increasing sequence of eigenvalues $\left(\lambda_{n}\right)_{n \in \mathbb{Z}}$ with no finite accumulation point, and if $\delta_{\lambda}$ denotes the point evaluation vector in $\mathcal{H}$ at any $\lambda \in \mathbb{R}$, then $\left\{\delta_{\lambda_{n}}\right\}$ is a total orthogonal set in $\mathcal{H}$.

The main goal of this paper is to fully connect the theory developed in [4-6] with the theory of de Branges spaces by showing that any linear transformation $B$ with domain and range contained in a separable Hilbert space $\mathcal{H}$ is regular, closed and simple symmetric with deficiency indices $(1,1)$ if and only if it is unitarily equivalent to multiplication by the independent variable in a de Branges space of entire functions. ${ }^{1}$ This will be accomplished by straightforwardly combining known results about de Branges-Rovnyak spaces and Lifschitz' theory of the characteristic functions of simple symmetric operators with deficiency indices $(1,1)$ [10, Appendix 1], [11].

In the process of achieving this result, it will be demonstrated that multiplication by the independent variable is a simple symmetric linear transformation, with deficiency indices $(1,1)$ in any de Branges-Rovnyak space in the upper half plane provided this space is defined by an extreme point of the unit ball in $H^{\infty}$. New results on when this linear transformation is densely defined, and on the spectra of simple symmetric operators with deficiency indices $(1,1)$ will also be presented. Finally, new sufficient conditions for a subspace of $L^{2}(\mathbb{R}, d v)$ to be the image of a de Branges space of entire functions under an isometry which acts as multiplication by a measurable function will be proven (see Theorems 5.2.2 and 5.2.6 of Sect. 5.2).

\subsection{Some Notation}

We will define

$$
\mu(z):=\frac{z-i}{z+i} ; \quad \mu: \mathbb{U} \rightarrow \mathbb{D}
$$

so that $\mu^{-1}(z)=i \frac{1+z}{1-z}$. Here $\mathbb{U}$ denotes the open complex upper half plane, $\mathbb{D}$ the unit disc, and $\mathbb{T}, \mathbb{L}$ will be used for the unit circle and open complex lower half plane, respectively. Throughout this paper $\phi$ will denote a function in $B_{1}\left(H^{\infty}(\mathbb{U})\right)$, the unit ball of $H^{\infty}(\mathbb{U})$, and $\varphi:=\phi \circ \mu^{-1}$ will be the canonical image of $\phi$ in $B_{1}\left(H^{\infty}(\mathbb{D})\right), \phi=\varphi \circ \mu$. If $\phi$ is an extreme point of the unit ball of $H^{\infty}(\mathbb{U})$ we will sometimes simply say that $\phi$ is extreme. Let $M$ denote the operator of multiplication by the independent variable in $L^{2}(\mathbb{R})$, so that $\phi(M)$ is multiplication by $\phi$. Let $\mathcal{U}: H^{2}(\mathbb{D}) \rightarrow H^{2}(\mathbb{U})$ denote the canonical isometry of $H^{2}(\mathbb{D})$ onto $H^{2}(\mathbb{U})$. If $f \in H^{2}(\mathbb{D})$,

$$
\mathcal{U} f(z)=\frac{1-\mu(z)}{\sqrt{\pi}} f(\mu(z)) \in H^{2}(\mathbb{U})
$$

\footnotetext{
1 As observed in [4,9] if $B$ is actually an entire operator in the sense of Krein [4,8], then it is already known that the space $\mathcal{H}$ described in the previous paragraph is a de Branges space.
} 
and if $f \in H^{2}(\mathbb{U})$, then

$$
\mathcal{U}^{-1} f(z)=\sqrt{\pi} \frac{f\left(\mu^{-1}(z)\right)}{1-z} \in H^{2}(\mathbb{D})
$$

It follows that $\mathcal{U}^{-1} \phi(M) \mathcal{U}$ acts as multiplication by $\varphi$ on $H^{2}(\mathbb{D})$.

For $f \in L^{\infty}(\mathbb{R})$, let $T_{f}:=\left.P_{H^{2}(\mathbb{U})} f(M)\right|_{H^{2}(\mathbb{U})}$, where $f(M)$ is multiplication by $f$. The notation $K_{\phi}^{2}$ will be used for the de Branges-Rovnyak space which is the range of $R_{\phi}:=\sqrt{1-T_{\phi} T_{\bar{\phi}}}$, endowed with the inner product that makes $R_{\phi}$ a co-isometry of $H^{2}(\mathbb{U})$ onto its range. Hence if $f, g \in H^{2}(\mathbb{U})$ and at least one of them is orthogonal to $\operatorname{Ker}\left(R_{\phi}\right)$, the kernel of $R_{\phi}$, then $\left\langle R_{\phi} f, R_{\phi} g\right\rangle_{\phi}=\langle f, g\rangle$, where $\langle\cdot, \cdot\rangle_{\phi}$ denotes the inner product in $K_{\phi}^{2}$. The notation $\mathcal{H}(E)$ is reserved for the de Branges space of entire functions defined using the de Branges function $E$.

It is not hard to check that $\mathcal{U}$ is an isometry of $K_{\phi}^{2}$ onto $K_{\varphi}^{2}$, where $K_{\varphi}^{2}$ is the usual de Branges-Rovnyak space of functions in $H^{2}(\mathbb{D})$, defined using $\varphi$, i.e., $K_{\varphi}^{2}$ is the range of $\hat{R}_{\varphi}:=\sqrt{1-\hat{T}_{\varphi} \hat{T}_{\bar{\varphi}}}$ in $H^{2}(\mathbb{D})$ endowed with the inner product that makes $\hat{R}_{\varphi}$ a co-isometry of $H^{2}(\mathbb{D})$ onto $K_{\varphi}^{2}$. Here $\hat{T}_{\varphi}$ is the Toeplitz operator $\left.P_{H^{2}(\mathbb{D})} \hat{M}_{\varphi}\right|_{H^{2}(\mathbb{D})}$, and $\hat{M}_{\varphi}=\mathcal{U}^{-1} \phi(M) \mathcal{U}$ acts as multiplication by $\varphi$ on $H^{2}(\mathbb{D})$. The notation, $\langle\cdot, \cdot\rangle_{\phi}$ and $\langle\cdot, \cdot\rangle_{\varphi}$ will be used for the inner products in $K_{\phi}^{2}$ and $K_{\varphi}^{2}$, respectively. Of course these inner products reduce to the usual $L^{2}$ inner products in the case where $\phi=F$ is an inner function. If it is clear from the context which space is being dealt with, the generic $\langle\cdot, \cdot\rangle$ will sometimes be used. All inner products are assumed to be conjugate linear in the second argument.

For future reference, for $w \in \mathbb{U}$, we will let $k_{w}^{\phi}$ denote the point evaluation vector at $w$ in $K_{\phi}^{2}$. That is $k_{w}^{\phi}$ is the element of $K_{\phi}^{2}$ such that $\left\langle f, k_{w}^{\phi}\right\rangle_{\phi}=f(w)$ for all $f \in K_{\phi}^{2}$. This vector has the form

$$
k_{w}^{\phi}(z):=\frac{i}{2 \pi} \frac{1-\overline{\phi(w)} \phi(z)}{z-\bar{w}} .
$$

Similarly, for $w \in \mathbb{D}, k_{w}^{\varphi}$ will denote the point evaluation vector in $K_{\varphi}^{2}$ at $w$, given by the formula

$$
k_{w}^{\varphi}(z):=\frac{1-\overline{\varphi(w)} \varphi(z)}{1-\bar{w} z} .
$$

Finally for any function $f$, analytic on a region $\Omega, f^{*}$ will denote the function $f^{*}(z)=$ $\overline{f(\bar{z})}$ analytic in the reflection of the region $\Omega$ in the real axis. 


\section{Representation of an Arbitrary Simple Symmetric Linear Transformation with Deficiency Indices $(1,1)$}

Given a Hilbert space $\mathcal{H}$, let $V$ denote an arbitrary closed simple isometric linear transformation with deficiency indices $(1,1)$ in $\mathcal{H}$. Here, recall that the deficiency indices $\left(n_{+}, n_{-}\right)$of $V$ are defined as $n_{+}:=\operatorname{dim}\left(\operatorname{Dom}(V)^{\perp}\right)$ and $n_{-}:=\operatorname{dim}\left(\operatorname{Ran}(V)^{\perp}\right)$, and that an isometric linear transformation is called simple if it has no unitary restriction to any non-trivial subspace. Further recall that a linear transformation in $\mathcal{H}$ is called closed if its graph is a closed subspace of $\mathcal{H} \oplus \mathcal{H}$. An isometric linear transformation $V$ is bounded, and so will be closed provided its domain is a closed subspace of $\mathcal{H}$.

Given such a $V$, let $B:=\mu^{-1}(V)=i(1+V)(1-V)^{-1}$, where $(1-V)^{-1}$ is defined as a linear map from $\operatorname{Ran}(V-1)$ onto $\operatorname{Dom}(V)$. The map $B$ is a closed simple symmetric linear transformation in $\mathcal{H}$ with deficiency indices $(1,1)$. Recall that a symmetric linear transformation is called simple if it has no self-adjoint restriction to a dense domain in a non-trivial subspace, and that the deficiency indices of $B$ are also $\left(n_{+}, n_{-}\right)$where $n_{+}=\operatorname{dim}\left(\operatorname{Dom}(V)^{\perp}\right)=\operatorname{dim}\left(\operatorname{Ran}(B+i)^{\perp}\right)$ and $n_{-}=$ $\operatorname{dim}\left(\operatorname{Ran}(V)^{\perp}\right)=\operatorname{dim}\left(\operatorname{Ran}(B-i)^{\perp}\right)$. Note that if $B$ is densely defined, then its adjoint $B^{*}$ is a well-defined closed operator and $\operatorname{Ran}(B-\bar{z})^{\perp}=\operatorname{Ker}\left(B^{*}-z\right)$ for all $z \in \mathbb{C}$. Finally recall that $V=\mu(B)$, and that the map $B \mapsto \mu(B)$ is a bijection of the set of simple symmetric linear transformations with deficiency indices $(1,1)$ onto the set of simple isometric linear transformations with deficiency indices $(1,1)$. If $B$ is symmetric, $V:=\mu(B)$ is called its Cayley transform. For the basic theory on symmetric operators, their Cayley transforms and deficiency indices, see for example [10] or [12].

The Lifschitz characteristic function $w_{V}$ of a simple isometric linear transformation $V$ with deficiency indices $(1,1)$ is defined as

$$
w_{V}(z):=\frac{z\left\langle(U-z)^{-1} \psi_{+}, \psi_{+}\right\rangle}{\left\langle(U-z)^{-1} U \psi_{+}, \psi_{+}\right\rangle},
$$

where $U$ is an arbitrary unitary extension of $V$, and $0 \neq \psi_{+} \in \operatorname{Dom}(V)^{\perp}[10$, Appendix 1] [11]. Choosing a different unitary extension $U^{\prime}$ in the definition of $w_{V}$ only changes $w_{V}$ by a unimodular constant. For this reason $w_{V}$ is defined only up to such a constant, and we will say any two characteristic functions coincide if they differ by such a constant. The function $w_{V}$ belongs to $B_{1}\left(H^{\infty}(\mathbb{D})\right)$, and obeys $w_{V}(0)=0$.

The Lifschitz characteristic function of a simple symmetric linear transformation with deficiency indices $(1,1), B$, is then defined as $\omega_{B}(\lambda):=w_{V}(\mu(\lambda))$. A short calculation shows that for $a, \lambda \in \mathbb{C} \backslash\{-i\}$,

$$
\begin{aligned}
(\mu(a)-\mu(\lambda))^{-1} & =\left[\frac{1}{\lambda+i} \frac{a-i}{a+i}\left((\lambda+i)-(\lambda-i) \frac{a+i}{a-i}\right)\right]^{-1} \\
& =\frac{\lambda+i}{2 i} \frac{a+i}{a-i}\left(1+\frac{\lambda-i}{a-\lambda}\right) .
\end{aligned}
$$


If $U$ is a unitary extension of $V=\mu(B)$, then $A:=\mu^{-1}(U)$ is a self-adjoint extension of $B$.

It follows that the characteristic function of $B$ can be written as

$$
\omega_{B}(\lambda)=w_{\mu(B)}(\mu(\lambda))=\frac{\lambda-i}{\lambda+i} \frac{\left\langle\left(I+(\lambda-i)(A-\lambda)^{-1}\right) \psi_{+}, \mu(A) \psi_{+}\right\rangle}{\left\langle\left(I+(\lambda-i)(A-\lambda)^{-1}\right) \psi_{+}, \psi_{+}\right\rangle} .
$$

Again, $\omega_{B}$ is defined modulo unimodular constants, $\omega_{B} \in B_{1}\left(H^{\infty}(\mathbb{U})\right)$, and $\omega_{B}(i)=0$. Alternatively, one can calculate that $(\mu(a)-\mu(\lambda))^{-1}=\frac{\lambda+i}{2 i}\left(1+(\lambda+i)(a-\lambda)^{-1}\right)$, so that the characteristic function can also be written as

$$
\omega_{B}(\lambda)=\frac{\lambda-i}{\lambda+i} \frac{\left\langle\left(I+(\lambda+i)(A-\lambda)^{-1}\right) \psi_{+}, \psi_{+}\right\rangle}{\left\langle\left(I+(\lambda-i)(A-\lambda)^{-1}\right) \psi_{+}, \psi_{+}\right\rangle} .
$$

Remark 2.0.1 There is a slight technicality, glossed over in the above discussion that should be pointed out. It is straightforward to show that $B$ is densely defined if and only if no unitary extension $U$ of the Cayley transform $V=\mu(B)$ of $B$ has 1 as an eigenvalue (see the last part of the proof of Theorem 3.1.2 to come). Hence if $B$ is not densely defined, then there is a unitary extension $U_{1}$ of $V$ which has 1 as an eigenvalue so that for this particular $U_{1}, A=\mu^{-1}\left(U_{1}\right)$ is not well defined. This unitary extension is unique, if any other unitary extension $U^{\prime}$ also has 1 as an eigenvalue then one can calculate that 1 would have to be an eigenvalue of $V$, contradicting the simplicity of $V$. In conclusion, if $B$ is not densely defined, then in the formula (2.3) for the characteristic function of $B, A=\mu^{-1}(U)$ must be chosen as the inverse Cayley transform of a unitary extension $U$ of $V=\mu(B)$ where $U \neq U_{1}$.

We will employ the following theorems of M. S. Lifschitz on such isometric and symmetric linear transformations and their characteristic functions [10, Appendix 1] [11]:

Theorem 2.0.2 (Lifschitz) In order that two simple isometric linear transformations with deficiency indices $(1,1)$ be unitarily equivalent, it is necessary and sufficient that their characteristic functions coincide.

Theorem 2.0.3 (Lifschitz) Given any $w \in B_{1}\left(H^{\infty}(\mathbb{D})\right)$ obeying $w(0)=0$, there exists a simple isometric linear transformation with deficiency indices $(1,1)$ whose characteristic function is $w$.

Remark 2.0.4 Using the bijective correspondence between simple isometric and symmetric linear transformations with deficiency indices $(1,1)$, the analogous statements obtained by replacing $w \in B_{1}\left(H^{\infty}(\mathbb{D})\right)$ with $\omega \in B_{1}\left(H^{\infty}(\mathbb{U})\right)$, isometric with symmetric, and $w(0)=0$ with $\omega(i)=0$ in the above two theorems are also true.

Let $\varphi \in B_{1}\left(H^{\infty}(\mathbb{D})\right)$, and consider the function $b_{\varphi}:=\frac{1+\varphi}{1-\varphi}$. Then $b_{\varphi}$ has nonnegative real part, and so has the Herglotz integral representation

$$
b_{\varphi}(z)=\int_{0}^{2 \pi} \frac{e^{i \theta}+z}{e^{i \theta}-z} d \rho_{\varphi}\left(e^{i \theta}\right)+i \operatorname{Im}\left(b_{\varphi}(0)\right)
$$


where $\rho_{\varphi}$ is a positive, finite Borel measure on the unit circle $\mathbb{T}$ and $\operatorname{Im}(z)$ denotes the imaginary part of $z$. Now consider the space $L_{\varphi}^{2}(\mathbb{T})$ of functions on the unit circle square integrable with respect to this measure $\rho_{\varphi}$, and let $Z_{\varphi}$ denote the unitary operator of multiplication by $z$ in $L_{\varphi}^{2}(\mathbb{T})$, i.e. $Z_{\varphi} f\left(e^{i \theta}\right)=e^{i \theta} f\left(e^{i \theta}\right)$. Let $\psi_{+} \in L_{\varphi}^{2}(\mathbb{T})$ be defined by $\psi_{+}(z):=1 / z$. If $\operatorname{Dom}\left(Z_{\varphi}^{\prime}\right):=\left\{f \in L_{\varphi}^{2}(\mathbb{T}) \mid\left\langle f, \phi_{+}\right\rangle=0\right\}$, then it is not hard to check that $Z_{\varphi}^{\prime}:=\left.Z_{\varphi}\right|_{\operatorname{Dom}\left(Z_{\varphi}^{\prime}\right)}$ is a closed simple isometric linear transformation with deficiency indices $(1,1)$ whose characteristic function is

$$
w_{\varphi}(z)=\frac{\varphi(z)-\varphi(0)}{1-\overline{\varphi(0)} \varphi(z)} .
$$

In particular, if $\varphi(0)=0$ then $w_{\varphi}(z)=\varphi(z)$. These facts are collected in the following lemma.

Lemma 2.0.5 Let $V$ be a simple isometric linear transformation with deficiency indices $(1,1)$ and characteristic function $w$. Then $V$ is unitarily equivalent to $Z_{w}^{\prime}$ : $\operatorname{Dom}\left(Z_{w}^{\prime}\right) \rightarrow L_{w}^{2}(\mathbb{T})$, where $Z_{w}^{\prime}$ acts as multiplication by $z$ on its domain. If $\varphi \in B_{1}\left(H^{\infty}(\mathbb{D})\right)$, then the characteristic function $w_{\varphi}$ of $Z_{\varphi}^{\prime}$ is given by Eq. (2.6).

The following proof of this lemma follows immediately from that of Theorem 2.0.3 in [10, Appendix 1].

Proof The final statement will be proven first. Suppose that $\varphi \in B_{1}\left(H^{\infty}(\mathbb{D})\right)$. By definition (see (2.1)), the characteristic function $v(z)$ of $Z_{\varphi}^{\prime}$ is equal to

$$
v(z)=\frac{z\left\langle\left(Z_{\varphi}-z\right)^{-1} \psi_{+}, \psi_{+}\right\rangle}{\left\langle\left(Z_{\varphi}-z\right)^{-1} Z_{\varphi} \psi_{+}, \psi_{+}\right\rangle},
$$

where $\psi_{+}(z)=1 / z$ in $L_{\varphi}^{2}(\mathbb{T})$. First observe that

$$
\left\langle\left(Z_{\varphi}-z\right)^{-1} \psi_{+}, \psi_{+}\right\rangle=\int_{0}^{2 \pi} \frac{1}{e^{i \theta}-z} d \rho_{\varphi}\left(e^{i \theta}\right),
$$

while

$$
\left\langle\left(Z_{\varphi}-z\right)^{-1} Z_{\varphi} \psi_{+}, \psi_{+}\right\rangle=\int_{0}^{2 \pi} \frac{e^{i \theta}}{e^{i \theta}-z} d \rho_{\varphi}\left(e^{i \theta}\right) .
$$


By the Herglotz representation (2.5) of $b_{\varphi}, \rho_{\varphi}(\{\mathbb{T}\})=\operatorname{Re}\left(b_{\varphi}(0)\right)$ and

$$
\begin{aligned}
b_{\varphi}(z)-b_{\varphi}(0) & =\int_{0}^{2 \pi} \frac{e^{i \theta}+z}{e^{i \theta}-z} d \rho_{\varphi}\left(e^{i \theta}\right)-\operatorname{Re}\left(b_{\varphi}(0)\right) \\
& =\int_{0}^{2 \pi} \frac{e^{i \theta}+z-\left(e^{i \theta}-z\right)}{e^{i \theta}-z} d \rho_{\varphi}\left(e^{i \theta}\right) \\
& =2 z\left\langle\left(Z_{\varphi}-z\right)^{-1} \psi_{+}, \psi_{+}\right\rangle .
\end{aligned}
$$

Similarly,

$$
b_{\varphi}(z)+\overline{b_{\varphi}(0)}=2\left\langle\left(Z_{\varphi}-z\right)^{-1} Z_{\varphi} \psi_{+}, \psi_{+}\right\rangle
$$

Using that $b_{\varphi}=\frac{1+\varphi}{1-\varphi}$, we conclude that

$$
v=\frac{b_{\varphi}-b(0)}{b_{\varphi}+\overline{b(0)}}=\chi \frac{\varphi-\varphi(0)}{1-\overline{\varphi(0)} \varphi}
$$

where $\chi=\frac{1-\overline{\varphi(0)}}{1-\varphi(0)} \in \mathbb{T}$. This establishes the formula (2.6), and the final statement of the lemma.

To prove the remainder of the lemma, recall that if $w$ is the characteristic function of $V$, then $w(0)=0$. By (2.6), the characteristic function of $Z_{w}^{\prime}$ is also $w$. The rest of the lemma now follows from Theorem 2.0.2.

\subsection{An Isometry of $K_{\varphi}^{2}$ onto $H_{\varphi}^{2}$}

Let $\rho_{\varphi}$ be the positive Borel measure on the circle $\mathbb{T}$ defined by the function $\varphi \in B_{1}\left(H^{\infty}(\mathbb{D})\right)$ as in Eq. (2.5). The Cauchy integral of $\rho_{\varphi}$ is defined by

$$
\Gamma_{\varphi}(z)=\int_{\mathbb{T}} \frac{1}{1-\bar{w} z} d \rho_{\varphi}(w),
$$

[13, Chapter III]. Given $f \in L_{\varphi}^{2}(\mathbb{T})$, one defines $\Gamma_{\varphi} f:=\int_{\mathbb{T}} \frac{f(w)}{1-\bar{w} z} d \rho_{\varphi}(w)$. Let $H_{\varphi}^{2}$ denote the closure of the polynomials in $L_{\varphi}^{2}$. For any $f \in H_{\varphi}^{2}$, define $W_{\varphi} f(z):=$ $(1-\varphi(z)) \Gamma_{\varphi} f(z)$. Then as shown, for example in [13, III-7], $W_{\varphi}$ is an isometry of $H_{\varphi}^{2}$ onto $K_{\varphi}^{2}$.

It follows from [13, III-8] that

$$
W_{\varphi}\left(\left.Z_{\varphi}\right|_{H_{\varphi}^{2}}\right)^{*} W_{\varphi}^{-1}=X+(1-\varphi(0))^{-1}\left\langle\cdot, k_{0}^{\varphi}\right\rangle_{\varphi} S^{*} \varphi
$$


where $S^{*}$ is the backward shift in $H^{2}(\mathbb{D})$ and $X:=\left.S^{*}\right|_{K_{\varphi}^{2}}$. Furthermore, as shown in [13, III-7], the image of the constant function $f(z)=1$ under $W_{\varphi}$ is $(1-\varphi(0))^{-1} k_{0}^{\varphi}$.

\subsection{The Case Where $\varphi$ is Extreme}

Recall that $\varphi$ is an extreme point of the unit ball if and only if $\ln (1-|\varphi|)$ is not integrable [14, pg. 138]. If $H_{\varphi}^{2}$ is the closure of the polynomials in $L_{\varphi}^{2}$, further recall that $H_{\varphi}^{2}=L_{\varphi}^{2}$ if and only if the Radon-Nikodym derivative of the absolutely continuous part of $\rho_{\varphi}$ with respect to Lebesgue measure is not log-integrable [14, pg. 50]. This derivative is equal to $\frac{1-|\varphi|^{2}}{|1-\varphi|^{2}}$ and hence is not log-integrable if and only if $\varphi$ is an extreme point.

Since $Z_{\varphi}^{*}$ agrees with $\left(Z_{\varphi}^{\prime}\right)^{*}$ on $\operatorname{Ran}\left(Z_{\varphi}^{\prime}\right), \operatorname{Ran}\left(Z_{\varphi}^{\prime}\right)$ is the orthogonal complement of the constant function 1 in $L_{\varphi}^{2}$, and the image of 1 under the isometry $W_{\varphi}$ is a constant multiple of $k_{0}^{\varphi}$, it follows that the image of $\operatorname{Ran}\left(Z_{\varphi}^{\prime}\right)$ under the isometry $W_{\varphi}$ is the subspace $\mathcal{S}_{0} \subset K_{\varphi}^{2}$ of functions in $K_{\varphi}^{2}$ which vanish at 0 . It then follows from Eq. (2.14) that the image of $\left(Z_{\varphi}^{\prime}\right)^{*}$ under $W_{\varphi}$ is $\left.S^{*}\right|_{\mathcal{S}_{0}}$.

If $f(z)=z g(z) \in \mathcal{S}_{0}$, then it is clear that $g=S^{*} f \in K_{\varphi}^{2}$ since $K_{\varphi}^{2}$ is invariant under $S^{*}$. Moreover, by [13, II-9], with $X=\left.S^{*}\right|_{K_{\varphi}^{2}}$,

$$
X^{*} X f=X^{*} S^{*} f=S S^{*} f-\left\langle S^{*} f, S^{*} \varphi\right\rangle_{\varphi} \varphi=f-\left\langle S^{*} f, S^{*} \varphi\right\rangle_{\varphi} \varphi .
$$

Since $\varphi$ is an extreme point, it follows from [13, V-3], that $\varphi \notin K_{\varphi}^{2}$. Hence it must be that the inner product $\left\langle S^{*} f, S^{*} \varphi\right\rangle_{\varphi}=0$, that $X^{*} X f=S S^{*} f=f$ and that $\left.X^{*}\right|_{S^{*} \mathcal{S}_{0}}=\left.S\right|_{S^{*} \mathcal{S}_{0}}$. It follows that $\left.S\right|_{S^{*} \mathcal{S}_{0}}=W_{\varphi} Z_{\varphi}^{\prime} W_{\varphi}^{-1}$ is an isometry from the subspace $S^{*} \mathcal{S}_{0}$ onto $\mathcal{S}_{0}$.

The image of $S$ under the canonical isometry, $\mathcal{U}$, of $H^{2}(\mathbb{D})$ onto $H^{2}(\mathbb{U})$ is $\mu(M)$, which acts as multiplication by $\mu(z)=\frac{z-i}{z+i}$. The image of $\mathcal{S}_{0}$ under $\mathcal{U}$ is the subspace $\mathcal{S}_{i} \subset K_{\phi}^{2}$ of functions in $K_{\phi}^{2}$ which vanish at $z=i$. It follows that if $V^{\phi}:=$ $\mathcal{U} S \mathcal{U}^{-1}\left|\mathcal{U} S^{*} \mathcal{S}_{0}=\mathcal{U} W_{\varphi} Z_{\varphi}^{\prime} W_{\varphi}^{*} \mathcal{U}^{*}\right|_{1 / \mu(M) \mathcal{S}_{i}=\mathcal{U} W_{\varphi} \operatorname{Dom}\left(Z_{\varphi}^{\prime}\right)}$, then $\left(V^{\phi}\right)^{*}=1 /\left.\mu(M)\right|_{\mathcal{S}_{i}}$ where $1 / \mu(M)$ is multiplication by $1 / \mu$, and $V^{\phi}=\left.\mu(M)\right|_{\operatorname{Ran}\left(\left(V_{\phi}\right)^{*}\right)}$ is the image of $Z_{\varphi}^{\prime}$ under the isometry $\mathcal{U} W_{\varphi}$. Define the linear transformation $\left(V^{\phi}-1\right)^{-1}$ : $\operatorname{Ran}\left(V^{\phi}-1\right) \rightarrow \operatorname{Dom}\left(V^{\phi}\right)$. Then the inverse Cayley transform of $V^{\phi}, M^{\phi}:=$ $\mu^{-1}\left(V^{\phi}\right)=i\left(1+V^{\phi}\right)\left(1-V^{\phi}\right)^{-1}$, is a symmetric linear transformation which acts as multiplication by $z$ on the domain $\operatorname{Dom}\left(M^{\phi}\right):=\operatorname{Ran}\left(V^{\phi}-1\right) \subset K_{\phi}^{2}$, $M^{\phi}=\left.M\right|_{\operatorname{Dom}\left(M^{\phi}\right)}$. This domain will be dense if and only if $\operatorname{Ran}\left(V^{\phi}-1\right)$ is dense in $K_{\phi}^{2}$. We will provide necessary and sufficient conditions on $\phi$ for $M^{\phi}$ to be a densely defined symmetric operator in $K_{\phi}^{2}$ in the case where $\phi$ is extreme in Sect. 3.

Applying Lemma 2.0.5 and Remark 2.0.4 immediately yields:

Theorem 2.2.1 The transformation $V^{\phi}:=\left.\mu(M)\right|_{1 / \mu(M) \mathcal{S}_{i}}$ is a closed simple isometric transformation with deficiency indices $(1,1)$ in $K_{\phi}^{2}$. Its inverse Cayley transform, $M^{\phi}=\mu^{-1}\left(V^{\phi}\right)$ is a closed simple symmetric linear transformation with deficiency 
indices $(1,1)$ which acts as multiplication by $z$ on the domain $\operatorname{Dom}\left(M^{\varphi}\right)=$ $\operatorname{Ran}\left(V^{\varphi}-1\right) \subset K_{\varphi}^{2}$. The characteristic function of $M^{\phi}$ is

$$
\omega_{\phi}=\frac{\phi-\phi(i)}{1-\overline{\phi(i)} \phi}=w_{\varphi} \circ \mu
$$

Conversely, if $B$ is any closed simple symmetric linear transformation with deficiency indices $(1,1)$ whose characteristic function $\omega$ is an extreme point, then $B$ is unitarily equivalent to $M^{\phi_{\alpha}}$ in the de Branges-Rovnyak space $K_{\phi_{\alpha}}^{2}$ where $\phi_{\alpha}=\frac{\omega-\alpha}{1-\bar{\alpha} \omega}$ and $\alpha=-\phi_{\alpha}(i) \in \mathbb{D}$ is arbitrary.

Again, as observed in Remark 2.0.4, an analogous statement to the second part of the above theorem holds for any simple isometric linear transformation with indices $(1,1)$.

Remark 2.2.2 In particular, choosing $\alpha=0$ in the above theorem shows that $B$ is unitarily equivalent to multiplication by $z$ in $K_{\omega}^{2}$. It is easy to check that $\phi$ is inner or extreme if and only if $\omega$ is.

Remark 2.2.3 If $M$ denotes multiplication by $x$ in $L^{2}(\mathbb{R})$ and $F$ is an inner function, it is not difficult to verify that $M^{F}$ is the unique closed symmetric restriction of $M$ to a linear subspace of $K_{F}^{2}$ with deficiency indices $(1,1)$.

\subsubsection{A Conjugation which Commutes with $M^{\phi}$}

In the case where $\varphi$ is extreme, so that $W_{\varphi}$ is an isometry of $L_{\varphi}^{2}$ onto $K_{\varphi}^{2}$, let $C$ denote the conjugation defined on $L_{\varphi}^{2}$ by $C f(z)=1 / z \overline{f(z)}$ (see for example [15]). Recall that a conjugation is an idempotent norm-preserving anti-linear map. It is easy to observe that $C Z_{\varphi}=Z_{\varphi}^{*} C$, that $C \operatorname{Dom}\left(Z_{\varphi}^{\prime}\right)=\operatorname{Ran}\left(Z_{\varphi}^{\prime}\right)$, and that $C \operatorname{Dom}\left(Z_{\varphi}^{\prime}\right)^{\perp}=\operatorname{Ran}\left(Z_{\varphi}^{\prime}\right)^{\perp}$. With these facts one can show that if $C_{\phi}$ denotes the image of $C$ in $K_{\phi}^{2}, C_{\phi}:=\mathcal{U} W_{\varphi} C W_{\varphi}^{*} \mathcal{U}^{*}$, that $C_{\phi} k_{\lambda}^{\phi}(z)=\frac{1}{2 \pi i} \frac{\phi(z)-\phi(i)}{z-i}$, and that the following lemma holds.

Lemma 2.2.5 The conjugation $C_{\phi}$ maps $\operatorname{Dom}\left(M^{\phi}\right)$ onto itself and commutes with $M^{\phi}$. For any $\lambda \in \mathbb{C} \backslash \mathbb{R}, C_{\phi} \operatorname{Ran}\left(M^{\phi}-\lambda\right)^{\perp}=\operatorname{Ran}\left(M^{\phi}-\bar{\lambda}\right)^{\perp}$.

Any $\phi \in B_{1}\left(H^{\infty}(\mathbb{U})\right)$ has the canonical representation

$$
\phi(z)=\chi \prod_{n=1}^{\infty} \frac{1-z / z_{n}}{1-z / \overline{z_{n}}} e^{i \sigma z} \exp \left(i \int_{-\infty}^{\infty} \frac{1+t z}{t-z} d v(t)\right)
$$

where $\left(z_{n}\right)_{n \in \mathbb{N}} \subset \mathbb{U}, \sum_{n}\left|\operatorname{Im}\left(\frac{1}{z_{n}}\right)\right|<\infty, \sigma \geq 0, \chi \in \mathbb{T}$ and $v$ is a finite, positive Borel measure on $\mathbb{R}$. This formula actually defines a function which is analytic everywhere in the region $\Omega$ where $\Omega:=\mathbb{C} \backslash \operatorname{supp}(\phi)^{*}$ and $\operatorname{supp}(\phi)^{*}$ is defined as the union of the closure of the set $\left\{\overline{z_{n}}\right\}_{n \in \mathbb{N}}$ with the closed support of the measure $v$ on $\mathbb{R}$. Recalling that $\phi^{*}$ is defined by $\phi^{*}(z)=\overline{\phi(\bar{z})}$, Eq. (2.17) implies that $\phi(z) \phi^{*}(z)=1$ for all $z \in \Omega$. 
It is clear that if $h \in H^{2}(\mathbb{U})$ then $h^{*} \in H^{2}(\mathbb{L})$, and visa versa. If $\phi=F$ is inner, so that $F(x) \overline{F(x)}=1$ almost everywhere $x \in \mathbb{R}$, then the non-tangential limits of $F$ as $z$ approaches the real axis from either the upper or lower half-planes are equal to $F(x)$ almost everywhere. To see this, note that if $z \in \mathbb{L}, F(z)=1 / F^{*}(z)$, and the non-tangential limits of $F(z)$ as $z$ approaches the real axis from below equal $1 / \overline{F(x)}=F(x)$ almost everywhere. Hence if $h \in H^{2}(\mathbb{U}), F h^{*}$ is that function whose non-tangential limits as $z$ approaches the real axis from below equal $F(x) \overline{h(x)}$ almost everywhere, and if $f, g \in H^{2}(\mathbb{U})$ then $\langle F f, g\rangle=\left\langle f, F^{*} g\right\rangle$. Let $*$ denote the operation $f \mapsto f^{*}$, and $F(M)$ be multiplication by $F$.

Claim 2.2.6 If $F$ is inner, the mapping $\widetilde{C}_{F}:=F(M) \circ *$ is a conjugation on $K_{F}^{2}=$ $H^{2}(\mathbb{U}) \ominus F H^{2}(\mathbb{U})$, and $f \in K_{F}^{2}$ if and only if both $f, F f^{*}$ belong to $H^{2}(\mathbb{U})$.

Proof Suppose $f \in K_{F}^{2}$. Then if $h \in H^{2}(\mathbb{U})$ is arbitrary, $\left\langle F^{*} f, h\right\rangle=\langle f, F h\rangle=0$ so that $F^{*} f \in L^{2}(\mathbb{R}) \ominus H^{2}(\mathbb{U})=H^{2}(\mathbb{L})$ and $\widetilde{C}_{F} f=F f^{*} \in H^{2}(\mathbb{U})$. Conversely, suppose that $f, F f^{*} \in H^{2}(\mathbb{U})$. Then given any $F h \in F H^{2}(\mathbb{U}),\langle f, F h\rangle=\left\langle F^{*} f, h\right\rangle=0$ since $F^{*} f \in L^{2}(\mathbb{R}) \ominus H^{2}(\mathbb{U})$. It follows that $f \in H^{2}(\mathbb{U}) \ominus F H^{2}(\mathbb{U})=K_{F}^{2}$.

The above shows that if $f \in K_{F}^{2}$, then so is $\widetilde{C}_{F} f$. The mapping $\widetilde{C}_{F}$ is clearly norm-preserving and anti-linear, so to show it is a conjugation, it remains to show that it is idempotent. If $f \in K_{F}^{2}$, then $\widetilde{C}_{F}^{2} f=F\left(F f^{*}\right)^{*}=F F^{*} f=f$.

More generally if $\phi \in B_{1}\left(H^{\infty}\right)$ is an extreme point, let $\widetilde{C}_{\phi}=\phi(M) \circ *$.

Claim 2.2.7 The map $\widetilde{C}_{\phi}$ defined on the linear span of the point evaluation vectors $k_{w}^{\phi}, w \in \mathbb{U}$ is anti-linear, norm-preserving and idempotent with range contained in $K_{\phi}^{2}$. Its unique continuous and anti-linear extension to all of $K_{\phi}^{2}$ is the conjugation $C_{\phi}$.

Proof Consider the dense linear manifold in $K_{\phi}^{2}$ which consists of all finite linear combinations of the point evaluation vectors $k_{w}^{\phi}$, for $w \in \mathbb{U}$. It follows that $\widetilde{C}_{\phi} k_{w}^{\phi}(z)=$ $\phi(z)\left(k_{w}^{\phi}\right)^{*}(z)=\phi(z) \frac{i}{2 \pi} \frac{1-\phi(w) \phi^{*}(z)}{w-z}=\frac{-i}{2 \pi} \frac{\phi(z)-\phi(w)}{z-w}$. As mentioned in Sect. 2.2.4 above, this is equal to $C_{\phi} k_{w}^{\phi}$, so that $\widetilde{C}_{\phi}$ and $C_{\phi}$ are norm preserving, and anti-linear maps that agree on a dense linear subspace of $K_{\phi}^{2}$. It follows that $\widetilde{C}_{\phi}$ can be extended by anti-linearity and continuity to all of $K_{\phi}^{2}$ and that this extension agrees with $C_{\phi}$.

\subsubsection{The Characteristic Function of $M^{\phi}$}

When $\phi$ is extreme, one can also directly calculate the characteristic function $\omega_{\phi}=$ $\omega_{M^{\phi}}$ of $M^{\phi}$ as follows. Consider the formula for the characteristic function $\omega_{B}$ of a simple symmetric linear transformation $B$ with deficiency indices $(1,1)$ as given in Eq. (2.3),

$$
\omega_{B}(\lambda):=w_{V}(\mu(\lambda))=\frac{\lambda-i}{\lambda+i} \frac{\left\langle\left(I+(\lambda-i)(A-\lambda)^{-1}\right) \psi_{+}, \mu(A) \psi_{+}\right\rangle}{\left\langle\left(I+(\lambda-i)(A-\lambda)^{-1}\right) \psi_{+}, \psi_{+}\right\rangle} .
$$

If $U$ is an arbitrary unitary extension of $\mu(B)$ which does not have 1 as an eigenvalue (see Remark 2.0.1), and $A=\mu^{-1}(U)$, it is not difficult to show that the operator 
$(A-z)\left(A-z^{\prime}\right)^{-1}=\left(I+\left(z-z^{\prime}\right)(A-z)^{-1}\right)$ maps $\operatorname{Ran}\left(B-\overline{z^{\prime}}\right)^{\perp}$ onto $\operatorname{Ran}(B-\bar{z})^{\perp}$ [8, pg. 9]. Consider $B=M^{\phi}$, the symmetric transformation of multiplication by $z$ in $K_{\phi}^{2}$, where $\phi$ is an extreme point of the unit ball in $H^{\infty}$. Observe that $k_{z}^{\phi} \in$ $\operatorname{Ran}\left(M^{\phi}-z\right)^{\perp}$ for all $z \in \mathbb{U}$. Indeed, for any $f \in \operatorname{Dom}\left(M^{\phi}\right),\left\langle\left(M^{\phi}-z\right) f, k_{z}^{\phi}\right\rangle_{\phi}=0$. Note that if $M^{\phi}$ is actually densely defined, then $\operatorname{Ran}\left(M^{\phi}-z\right)^{\perp}=\operatorname{Ker}\left(\left(M^{\phi}\right)^{*}-\bar{z}\right)$ so that each $k_{z}^{\phi}$ is an eigenvector to the adjoint of $M^{\phi}$ with eigenvalue $\bar{z}$. Let $\omega_{\phi}=\omega_{M^{\phi}}$, be defined using an arbitrary unitary extension $U$ of the Cayley transform of $M^{\phi}$, and let $M^{\prime}=A$ be the inverse Cayley transform of $U$. Since $\left(I+\left(z-z^{\prime}\right)\left(M^{\prime}-z\right)^{-1}\right) \psi_{+} \in$ $\operatorname{Ran}(M-\bar{z})^{\perp}, \operatorname{Ran}(M-\bar{\lambda})^{\perp}=\mathbb{C}\left\{k_{\lambda}\right\}$, the one dimensional subspace spanned by $k_{\lambda}$, and $\operatorname{Ran}(M-\bar{\lambda})^{\perp}=\mathbb{C}\left\{C_{\phi} k_{\lambda}\right\}$ for each $\lambda \in \mathbb{U}$ by Lemma 2.2.5, it follows that $\left(I+(\lambda-i)\left(M^{\prime}-\lambda\right)^{-1}\right) \psi_{+}=c(\lambda) C_{\phi} k_{\lambda}$. Here $c(\lambda) \in \mathbb{C}$. Moreover, we can choose $\psi_{-}=k_{i} /\left\|k_{i}\right\|$ and $\psi_{+}=C_{\phi} k_{i} /\left\|k_{i}\right\|$. It follows that

$$
\begin{aligned}
\omega_{\phi}(\lambda) & =\frac{\lambda-i}{\lambda+i} \frac{\left\langle c(\lambda) C_{\phi} k_{\lambda}, k_{i}\right\rangle}{\left\langle c(\lambda) C_{\phi} k_{\lambda}, C_{\phi} k_{i}\right\rangle} \\
& =\frac{\lambda-i}{\lambda+i} \frac{\left\langle C_{\phi} k_{\lambda}, k_{i}\right\rangle}{\left\langle k_{i}, k_{\lambda}\right\rangle}=\frac{\lambda-i}{\lambda+i} \frac{\left(C_{\phi} k_{\lambda}\right)(i)}{k_{i}(\lambda)} \\
& =\frac{\phi(i)-\phi(\lambda)}{1-\overline{\phi(i)} \phi(\lambda)}
\end{aligned}
$$

which is the same (up to a unimodular constant) as the formula for $\omega_{\phi}=\omega_{M^{\phi}}$ given in the statement of Theorem 2.2.1.

\section{Necessary and Sufficient Conditions for $M^{\phi}$ to be Densely Defined}

Given a simple symmetric linear transformation $B$ with deficiency indices $(1,1)$, the following theorem of Lifschitz provides a necessary and sufficient condition on the characteristic function $\omega$ of $B$ for $B$ to be densely defined [10, Appendix 1]:

Theorem 3.0.9 (Lifschitz) $B$ will be densely defined if and only if $\lim _{\lambda \rightarrow \infty} \lambda(\omega(\lambda)-$ $\left.e^{i \alpha}\right)=\infty ; 0<\epsilon \leq \arg \lambda \leq \pi-\epsilon$, for each $\alpha \in[0,2 \pi)$ and any fixed $\epsilon>0$.

Let $w:=\omega \circ \mu^{-1}$ be the characteristic function of $V:=\mu(B)$. It is not hard to see that the above necessary and sufficient condition for $B$ to be a densely defined operator is related to the existence of the angular derivative of $w$ at the point $z=1$.

\subsection{Angular Derivatives}

Given $b \in B_{1}\left(H^{\infty}(\mathbb{D})\right), b$ has the canonical factorization

$$
b(z)=\chi z^{n} \prod_{n} \frac{\left|a_{n}\right|}{a_{n}} \frac{a_{n}-z}{1-\overline{a_{n}} z} \exp \left(-\int_{\mathbb{T}} \frac{\zeta+z}{\zeta-z} d \rho(\zeta)\right),
$$

where $\sum_{n}\left(1-\left|a_{n}\right|\right)<\infty, \chi \in \mathbb{T}$ and $\rho$ is a positive Borel measure on $\mathbb{T}$. 
The function $b$ is said to have an angular derivative at $z \in \mathbb{T}$ in the sense of $\mathrm{Ca}$ rathéodory if the non-tangential limits of $b, b^{\prime}$ exist at $z$ and the non-tangential limit of $b$ at $z$ has unit modulus. Define

$$
A_{b}:=\left\{z \in \mathbb{T}\left|\sum_{n} \frac{1-\left|a_{n}\right|^{2}}{\left|z-a_{n}\right|^{2}}+\int_{\mathbb{T}}\right| \theta-\left.z\right|^{-2} d \rho(\theta)<\infty\right\} .
$$

The following theorem, taken from [16], is a combination of results of [13,17].

Theorem 3.1.1 (Carathéodory, Ahern and Clark, Sarason) Given $b \in B_{1}\left(H^{\infty}(\mathbb{D})\right)$, and $\gamma \in \mathbb{T}$, the following are equivalent:

(i) There exists a unimodular constant c such that $\frac{b(z)-c}{z-\gamma} \in K_{b}^{2}$.

(ii) $\gamma \in A_{b}$.

(iii) $\liminf _{z \rightarrow \gamma} \frac{1-|b(z)|}{1-|z|}<\infty$.

(iv) $b$ has an angular derivative in the sense of Carathéodory at $\gamma$.

(v) every $f \in K_{b}^{2}$ has a non-tangential limit at $\gamma$.

Furthermore, if any of the above conditions hold, $c=b(\gamma):=\lim _{r \rightarrow 1} b(r \gamma)$ is unique, and if $k_{\gamma}^{b}(z):=\frac{1-\overline{b(\gamma)} b(z)}{1-\bar{\gamma} z}$, then for all $f \in K_{b}^{2}, f(\gamma)=\left\langle f, k_{\gamma}^{b}\right\rangle_{b}$.

With the aid of the above theorem we can now prove the following:

Theorem 3.1.2 Let $V$ be a simple isometric linear transformation with deficiency indices $(1,1)$, and characteristic function $w_{V}$. Any point $z \in \mathbb{T}$ is not an eigenvalue of any unitary extension $U$ of $V$ if and only if the angular derivative of $w_{V}$ does not exist at $z$. The symmetric linear transformation $B=\mu^{-1}(V)$ will be densely defined if and only if the angular derivative of $w_{V}$ does not exist at $z=1$.

This proof of this theorem is almost immediately implied by the proof of the Theorem 3.0.9 of [10, Appendix 1]. We provide a sketch of the proof here for the reader's convenience. Recall that all unitary extensions of $V$ can be labeled by a single real parameter $\alpha \in[0,2 \pi)$,

$$
U(\alpha):=V \oplus e^{i \alpha}\left\langle\cdot, \psi_{+}\right\rangle \psi_{-}
$$

on $\mathcal{H}=\operatorname{Dom}(V) \oplus \operatorname{Dom}(V)^{\perp}$, where $\psi_{+} \in \operatorname{Dom}(V)^{\perp}$ and $\psi_{-} \in \operatorname{Ran}(V)^{\perp}$ are fixed non-zero vectors of the same norm. Further recall that the vector $\psi_{+}$is a generating vector for each $U(\alpha)$ [10, Section 81, Lemma 1].

Proof (sketch) Let $w=w_{V}$ and let $F(\alpha ; t):=\chi_{[0, t)}(U(\alpha)), t \in[0,2 \pi)$ be the spectral distribution function of the unitary operator $U(\alpha)$. Here $\chi_{\Omega}$ denotes the characteristic function of the Borel set $\Omega \subset[0,2 \pi]$. Since $\psi_{+}$is a generating vector for each $U(\alpha)$, it follows that $z^{\prime}=e^{i \beta} \in \mathbb{T}$ will be an eigenvalue for $U(\alpha)$ if and only if the distribution function $\sigma_{\alpha}(t):=\left\langle F(\alpha ; t) \psi_{+}, \psi_{+}\right\rangle$has a jump at $t=\beta$. However, as in the proof of Theorem 3.0.9, one can calculate that

$$
\frac{e^{i \alpha}}{e^{i \alpha}-w(z)}=\int_{0}^{2 \pi} \frac{1}{1-e^{-i t} z} d \sigma_{\alpha}(t)
$$


from which it follows that the value of the jump at $t=\beta$ is given by

$$
\lim _{z \rightarrow e^{i \beta}} \frac{\left(1-e^{-i \beta} z\right) e^{i \alpha}}{e^{i \alpha}-w(z)}
$$

where $z$ approaches $e^{i \beta}$ non-tangentially.

In conclusion, $z^{\prime} \in \mathbb{T}$ is not an eigenvalue of any unitary extension of $V$ if and only if

$$
\lim _{z \rightarrow z^{\prime}} \frac{z^{\prime}-z}{e^{i \alpha}-w(z)}=0
$$

for all $\alpha \in[0,2 \pi)$ whenever $z \rightarrow z^{\prime}$ non-tangentially. This will happen if and only if

$$
\lim _{z \rightarrow z^{\prime}}\left|\frac{w(z)-e^{i \alpha}}{z-z^{\prime}}\right|=\infty
$$

for all $\alpha \in[0,2 \pi$ ). If Eq. (3.7) holds, then by part (i) of Theorem 3.1.1, the angular derivative of $w$ at $z^{\prime}$ does not exist. Conversely, suppose that the angular derivative of $w$ does not exist at $z^{\prime}=e^{i \beta}$. Observe that

$$
\left|\frac{w(z)-e^{i \alpha}}{z-z^{\prime}}\right| \geq \frac{1-|w(z)|}{\left|z-z^{\prime}\right|}
$$

and since $z$ approaches $z^{\prime}$ non-tangentially, $\frac{1-|z|}{\left|z^{\prime}-z\right|}$ is bounded below in this limit. It follows that

$$
\lim _{z \rightarrow z^{\prime}}\left|\frac{w(z)-e^{i \alpha}}{z-z^{\prime}}\right| \geq C \liminf z \rightarrow z^{\prime} \frac{1-|w(z)|}{1-|z|}=\infty
$$

by part (iii) of Theorem 3.1.1.

It remains to show that $B=\mu^{-1}(V)$ will be densely defined if and only if $z=1$ is not an eigenvalue of any unitary extension of $V$. This part of the proof is taken directly from [10, Appendix 1]. First, if $B$ is densely defined, then $\operatorname{Ran}(V-1)$ and hence $\operatorname{Ran}(U-1)$ is dense for any unitary extension $U$ of $V$. It follows easily from this that no such $U$ has 1 as an eigenvalue. Conversely, assume that $B$ is not densely defined, so that there is a vector $\xi \in \mathcal{H}$ such that $\langle(V-1) \psi, \xi\rangle=0$ for all $\psi \in \operatorname{Dom}(V)$. As before choose $\psi_{ \pm}$such that $1=\left\|\psi_{ \pm}\right\|$and $\operatorname{Dom}(V)^{\perp}=\mathbb{C}\left\{\psi_{+}\right\}, \operatorname{Ran}(V)^{\perp}=\mathbb{C}\left\{\psi_{-}\right\}$, and for $\kappa \in \mathbb{C}$ define $U_{\kappa}$ on $\mathcal{H}=\operatorname{Dom}(V) \oplus \mathbb{C}\left\{\psi_{+}\right\}$by $U_{\kappa}=V \oplus \kappa\left\langle\cdot, \psi_{+}\right\rangle \psi_{-}$. Given any $\psi \in \mathcal{H}$, write $\psi=\psi_{V}+c \psi_{+}$where $\psi_{V} \in \operatorname{Dom}(V)$. Then,

$$
\left\langle\left(U_{\kappa}-1\right) \psi, \xi\right\rangle=\left\langle(V-1) \psi_{V}, \xi\right\rangle+c\left(\kappa\left\langle\psi_{-}, \xi\right\rangle-\left\langle\psi_{+}, \xi\right\rangle\right)
$$

Now $\left\langle\psi_{-}, \xi\right\rangle$ cannot be zero, as otherwise, there would exist a $\xi^{\prime} \in \operatorname{Dom}(V)$ such that $V \xi^{\prime}=\xi$. This would imply $0=\left\langle(V-1) \psi, V \xi^{\prime}\right\rangle=\left\langle\psi,(1-V) \xi^{\prime}\right\rangle$ for all 
$\psi \in \operatorname{Dom}(V)$ so that $(V-1) \xi^{\prime}=c^{\prime} \psi_{+}$. The fact that $\xi^{\prime} \perp \psi_{+}$and $\left\|V \xi^{\prime}\right\|=\left\|\xi^{\prime}\right\|$ would then imply that $V \xi^{\prime}=\xi^{\prime}$, contradicting the simplicity of $V$.

Since $\left\langle\psi_{-}, \xi\right\rangle \neq 0$, choose $\kappa=\frac{\left\langle\psi_{+}, \xi\right\rangle}{\left\langle\psi_{-}, \xi\right\rangle}$ in (3.10) to obtain that $\left\langle\left(U_{\kappa}-1\right) \psi, \xi\right\rangle=0$ for all $\psi \in \mathcal{H}$. This implies that $U_{\kappa}^{*} \xi=\xi$. It is straightforward to calculate that $U_{\kappa}^{*}=V^{*} \oplus \bar{\kappa}\left\langle\cdot, \psi_{-}\right\rangle \psi_{+}$on $\mathcal{H}=\operatorname{Ran}(V) \oplus \mathbb{C}\left\{\psi_{-}\right\}$. Hence, writing $\xi=\xi^{*}+d \psi_{-}$ where $\xi^{*} \in \operatorname{Ran}(V)$ and $d \in \mathbb{C}$, it follows that $U_{\kappa}^{*} \xi=V^{*} \xi^{*}+\bar{\kappa} d \psi_{+}$, and that

$$
\left\|\xi^{*}\right\|^{2}+|d|^{2}=\|\xi\|^{2}=\left\|U_{\kappa}^{*} \xi\right\|^{2}=\left\|V^{*} \xi^{*}\right\|^{2}+|\kappa|^{2}|d|^{2}=\left\|\xi^{*}\right\|^{2}+|\kappa|^{2}|d|^{2} .
$$

This shows that $|\kappa|=1$ so that $U_{\kappa}$ is the desired unitary extension of $V$ which has 1 as an eigenvalue.

The following corollary follows readily from the above theorem, and part (ii) of Theorem 3.1.1.

Corollary 3.1.3 Let $B$ be a simple symmetric linear transformation with deficiency indices $(1,1)$, and characteristic function $\omega$. Consider the canonical representation (2.17) of $\omega$. Then $B$ is densely defined if and only if either $\sigma>0$ or

$$
\sum_{n \in \mathbb{N}} \operatorname{Im}\left(z_{n}\right)+\int_{-\infty}^{\infty}|t+i|^{2} d v(t)=\infty
$$

If $B$ is densely defined then $\lambda \in \mathbb{R}$ is not an eigenvalue of any self-adjoint extension of $B$ if and only if

$$
\sum_{n \in \mathbb{N}} \frac{\operatorname{Im}\left(z_{n}\right)}{\left|\lambda-z_{n}\right|^{2}}+\int_{-\infty}^{\infty} \frac{|t+i|^{2}}{|\lambda-t|^{2}} d \nu(t)=\infty
$$

The proof of this corollary is a straightforward computation.

Proof By Theorem 3.1.2, $B$ is densely defined if and only if the angular derivative of $w_{V}$ at $\gamma=1$ does not exist. As before $V:=\mu(B)$. By part (ii) of Theorem 3.1.1 this happens if and only if $1=\gamma \notin A_{w_{V}}$, i.e. if and only if

$$
\sum \frac{1-\left|a_{n}\right|^{2}}{\left|\gamma-a_{n}\right|^{2}}+\int_{\mathbb{T}}|\zeta-\gamma|^{-2} d \rho(\zeta)=\infty
$$

where $\left\{a_{n}\right\}$ are the zeroes of $w_{V}$ in $\mathbb{D}$ and $\rho$ is the singular measure appearing in the singular part of $w_{V}$. Explicitly, $w_{V}(z)=\delta \mathfrak{B}_{V}(z) S_{V}(z)$ where $|\delta|=1$, the Blaschke part of $w_{V}$ is

$$
\mathfrak{B}_{V}(z)=\prod_{n} \frac{\left|a_{n}\right|}{a_{n}} \frac{a_{n}-z}{1-\overline{a_{n}} z}
$$


and the singular part is

$$
S_{V}(z)=\exp \left(-\int_{\mathbb{T}} \frac{\zeta+z}{\zeta-z} d \rho(\zeta)\right)=e^{-\frac{1+z}{1-z} \rho(\{1\})} \exp \left(-\int_{\mathbb{T} \backslash\{1\}} \frac{\zeta+z}{\zeta-z} d \rho(\zeta)\right)
$$

The zeroes of $\omega_{B}$ are $z_{n}=\mu^{-1}\left(a_{n}\right) \in \mathbb{U}$. If we let $t=\mu^{-1}(\zeta)$ for $\zeta \in \mathbb{T} \backslash\{1\}$, $\sigma:=\rho(\{1\})$, and define the measure $\nu$ on $\mathbb{R}$ by $d \nu(t)=d \rho(\mu(t))$, then we see that the canonical representation of $\omega_{B}$ is $\omega_{B}(z)=\omega_{V}(\mu(z))=\delta \mathfrak{B}_{B}(z) S_{B}(z)$, where the singular part $S_{B}$ is

$$
S_{B}(z)=S_{V}(\mu(z))=e^{i \sigma z} \exp \left(i \int_{-\infty}^{\infty} \frac{1-t z}{t-z} d v(t)\right)
$$

and $\mathfrak{B}_{B}(z)=\mathfrak{B}_{V}(\mu(z))$ is a Blaschke product with zero set $\left\{z_{n}=\mu^{-1}\left(a_{n}\right)\right\}$.

A short calculation shows that if $\gamma=1$, then upon replacing $a_{n}=\mu\left(z_{n}\right)$, the summand appearing in (3.14) can be written $\frac{1-\left|a_{n}\right|^{2}}{\left|1-a_{n}\right|^{2}}=\frac{1}{4}\left(\left|z_{n}+i\right|^{2}-\left|z_{n}-i\right|^{2}\right)=\operatorname{Im}\left(z_{n}\right)$. Furthermore with $t, v$ and $\sigma$ as above, and $\gamma=1$ the integral appearing in (3.14) can be written

$$
\begin{aligned}
\int_{\mathbb{T}}|\zeta-1|^{-2} d \rho(\zeta) & =+\infty \cdot \sigma+\int_{\mathbb{T} \backslash\{1\}}|\zeta-1|^{-2} d \rho(\zeta) \\
& =+\infty \cdot \sigma+\int_{-\infty}^{\infty}|t+i|^{2} d \nu(t) .
\end{aligned}
$$

Here $+\infty \cdot \sigma$ is defined as $+\infty$ if the point mass $\sigma=\rho(\{1\})>0$ and 0 if $\sigma=0$. This proves the statement characterizing when $B$ is densely defined.

The second statement follows from similar calculations, and the observation that $\lambda \in \mathbb{R}$ is an eigenvalue of a self-adjoint extension of $B$ if and only if $\gamma=\mu(\lambda) \in \mathbb{T} \backslash\{1\}$ is an eigenvalue of some unitary extension of $V=\mu(B)$.

Corollary 3.1.4 The operator, $M^{\phi}$ of multiplication by $z$ in $K_{\phi}^{2}$ is densely defined if and only if the angular derivative of $\varphi=\phi \circ \mu^{-1}$ does not exist at $z=1$. If $M^{\phi}$ is densely defined, then $\lambda \in \mathbb{R}$ is not an eigenvalue of any of its self-adjoint extensions if and only if the angular derivative of $\varphi$ at $\mu(\lambda)$ does not exist.

Proof Using the formula for $w_{\varphi}$ given in Eq. (2.6), it is not difficult to verify that the angular derivative of $w_{\varphi}$ at $z \in \mathbb{T}$ exists if and only if the angular derivative of $\varphi$ exists at $z$. 


\section{Spectra of Self-Adjoint Extensions of $M^{\phi}$}

In this section we consider the case where $\phi$ is an extreme point, and $\phi$ obeys the conditions of Corollary 3.1.4 so that $M^{\phi}$, which acts as multiplication by $z$, is a closed, simple and densely defined symmetric linear operator with deficiency indices $(1,1)$ in $K_{\phi}^{2}$. The following result, due to Lifschitz, immediately identifies the essential spectrum of $M^{\phi}$ in $K_{\phi}^{2}$. Recall that a point $z \in \mathbb{C}$ is said to be a regular point, or a point of regular type for a closed linear transformation $T$ if $T-z$ is bounded below on $\operatorname{Dom}(T)$. A symmetric linear transformation $B$ is said to be regular if every $z \in \mathbb{C}$ is regular for $B$.

Theorem 4.0.5 (Lifschitz) In order that a real number $\lambda$ be a point of regular type of a simple symmetric linear transformation $B$ with deficiency indices $(1,1)$ and characteristic function $\omega$, it is necessary and sufficient that both of the following conditions be satisfied:

(1) $\omega$ is analytic in a neighbourhood of $\lambda$.

(2) $|\omega(t)|=1$ on some open interval of $\mathbb{R}$ containing $\lambda$.

In [18, Theorem 4], a more general version of the above theorem is established for simple isometric linear transformations (and hence for simple symmetric linear transformations) with deficiency indices $(n, n), n<\infty$.

\subsubsection{Essential Spectrum of $M^{\phi}$}

For a simple symmetric operator with deficiency indices $(1,1), \lambda$ belongs to the essential spectrum of $B$ if and only if $\lambda$ is not a regular point of $B$. For the operator of multiplication $M^{\phi}$, its characteristic function $\omega_{\phi}$ obeys the conditions of Theorem 4.0.5 at $\lambda \in \mathbb{R}$ if and only if $\phi$ obeys those same conditions. It follows that $\sigma_{e}\left(M^{\phi}\right)=\operatorname{supp}(\phi) \cap \mathbb{R} \cup\{\infty\}$ where $\operatorname{supp}(\phi)$ is defined as the union of the closure of the set of zeroes of $\phi$ and the closed support of the measure $v$ that appears in the canonical representation of $\phi$ in Eq. (2.17). This is clear as if $\lambda \in \mathbb{R}$ is either a limit point of the zeroes of $\phi$ or in the closed support of the part of the measure $v$ which is singular with respect to Lebesgue measure, then $\phi$ does not satisfy condition (1) of Theorem 4.0.5 at $\lambda$. Now suppose that $\lambda$ belongs to the closed support of the absolutely continuous part of $\nu$. By the inner-outer factorization for the $H^{\infty}$ function $\varphi=\phi \circ \mu^{-1}$, the absolutely continuous part of $\rho:=v \circ \mu^{-1}$ is given by $d \rho(\gamma)=-\ln |\varphi(\gamma)| d m(\gamma)$, where $m$ is normalized Lebesgue measure on $\mathbb{T}$. Since $\varphi \in B_{1}\left(H^{\infty}(\mathbb{D})\right)$, it follows that a Borel set $\Omega \subset \mathbb{R}$ belongs to the closed support of the absolutely continuous part of $v$ if and only if $|\phi(x)|<1$ almost everywhere for $x \in \Omega$. It follows that $\lambda$ belongs to the closure of a Borel subset $\Omega \subset \mathbb{R}$ of non-zero Lebesgue measure where $|\phi(x)|<1$ a.e. $x \in \Omega$ so that condition (2) of Theorem 4.0.5 implies that $\lambda \in \bar{\Omega} \subset \sigma_{e}\left(M^{\phi}\right)$.

If $A^{\prime}$ is any self-adjoint extension of a symmetric operator $A$ with finite deficiency indices, then $\sigma_{e}\left(A^{\prime}\right)=\sigma_{e}(A)$. This follows easily from the fact that $\mu\left(A^{\prime}\right)$ is a finite rank extension of $\mu(A)$. 
Remark 4.0.7 Note that since $M^{\phi}$ is simple, it has no eigenvalues so that $\sigma\left(M^{\phi}\right)=$ $\sigma_{e}\left(M^{\phi}\right)$. Further observe that if $\lambda \in \mathbb{R} \backslash \sigma_{e}\left(M^{\phi}\right)$, then by Theorem 4.0.5, the angular derivative of $\omega_{\phi}$ (and hence of $\phi$ ) exists at $\lambda$.

\subsubsection{Total Orthogonal Sets of Point Evaluation Vectors}

The spectra of self-adjoint extensions of $M^{\phi}$ are related to the existence of total orthogonal sets of point evaluation vectors in $K_{\phi}^{2}$. As discussed in [16], a set of point evaluation vectors $\Gamma:=\left\{k_{\lambda_{n}}^{\phi}\right\}_{n \in \mathbb{Z}}$ in $K_{\phi}^{2}$ can only be orthogonal if $\left\{\lambda_{n}\right\} \subset \mathbb{R}$. If $\Gamma$ is a total orthogonal set, the set of points $\left\{\lambda_{n}\right\}$ is the spectrum of a self-adjoint extension $M^{\prime}$ of $M^{\phi}$, and $\Gamma$ consists of eigenvectors $M^{\prime}$. To see this note that since $M^{\phi}$ is densely defined, each $k_{\lambda_{n}}^{\phi}$ is an eigenvector of $\left(M^{\phi}\right)^{*}$ with eigenvalue $\lambda_{n}$. Hence the closure, $M^{\prime}$ of the restriction of $\left(M^{\phi}\right)^{*}$ to the linear span of $\Gamma$ is a self -adjoint restriction of $\left(M^{\phi}\right)^{*}$. If $\psi \in \operatorname{Dom}(M)$, then $\left\langle\psi, M^{\prime} \psi^{\prime}\right\rangle=\left\langle\psi,\left(M^{\phi}\right)^{*} \psi^{\prime}\right\rangle=\left\langle M^{\phi} \psi, \psi^{\prime}\right\rangle$ for all $\psi^{\prime} \in \operatorname{Dom}\left(M^{\prime}\right)$. This implies that $\psi \in \operatorname{Dom}\left(\left(M^{\prime}\right)^{*}=M^{\prime}\right)$ and $\left(M^{\prime}\right)^{*} \psi=M^{\prime} \psi=$ $M^{\phi} \psi$, so that $\operatorname{Dom}\left(M^{\phi}\right) \subset \operatorname{Dom}\left(M^{\prime}\right)$, and $M^{\prime}$ is a self-adjoint extension of $M^{\phi}$.

In particular, Theorem 4.0.5 and Remark 4.0.7 allow one to conclude that if $K_{\phi}^{2}$ has a total orthogonal set of point evaluation vectors, then the angular derivatives of $\varphi$ must exist almost everywhere on $\mathbb{T}$ so that $\phi$ must be inner. This follows as if the angular derivative of $\varphi$ does not exist at a point $z \in \mathbb{T}$, then either $\varphi$ does not have unit modulus at $z$ or it is not analytic in any neighbourhood of $z$ so that $\mu^{-1}(z)$ belongs to the essential spectrum of $M^{\phi}$. If there is a Borel subset $\mu(\Omega) \subset \mathbb{T}$ of non-zero measure on which the angular derivatives of $\varphi$ do not exist, then $\Omega:=\mu^{-1}(\mu(\Omega)) \subset \mathbb{R}$ belongs to $\sigma_{e}\left(M^{\phi}\right)$ and no point of $\Omega$ belongs to the point spectrum of any self-adjoint extension of $M^{\phi}$ by Corollary 3.1.4. Hence if $M^{\prime}$ is any self-adjoint extension of $M^{\phi}$, and $\Lambda:=\left\{k_{\lambda_{n}}\right\}$ is a maximal set of orthogonal eigenvectors of $M^{\prime}$, then $\Omega \subset \sigma_{e}(M)=\sigma_{e}\left(M^{\prime}\right)$ and $\chi_{\Omega}\left(M^{\prime}\right)$ projects onto a non-zero subspace orthogonal to the closed linear span of $\Lambda$. Hence $K_{\phi}^{2}$ has no total orthogonal set of point evaluation vectors. This fact appears as Corollary 2.2 in [16].

\subsection{Spectra of Self-Adjoint Extensions of $M^{\phi}$}

Suppose that $\lambda \in \mathbb{R} \backslash \sigma_{e}\left(M^{\phi}\right)$. By Remark 4.0.7, the angular derivative of $\phi$ at $\mu(\lambda)$ exists, and it follows from Theorem 3.1.1 that point evaluation at $\lambda$ is a bounded linear functional on $K_{\phi}^{2}$, generated by inner products with the point evaluation vector

$$
k_{\lambda}^{\phi}(z):=\frac{i}{2 \pi} \frac{1-\overline{\phi(\lambda)} \phi(z)}{z-\lambda}
$$

The spectra of any fixed self-adjoint extension, $M^{\prime}$ of $M^{\phi}$ is $\sigma\left(M^{\prime}\right)=\sigma_{p}\left(M^{\prime}\right) \cup$ $\sigma_{e}\left(M^{\phi}\right)$ where $\sigma_{p}\left(M^{\prime}\right)$ denotes the set of eigenvalues of $M^{\prime}$. Note that if $\lambda \in \mathbb{R} \backslash$ $\sigma_{e}\left(M^{\phi}\right)$ is an eigenvalue of $M^{\prime}$, then it follows that $k_{\lambda}^{\phi}$ is an eigenvector of $M^{\prime}$ to eigenvalue $\lambda$. To see this, first note that since $M^{\phi}$ is densely defined, $k_{\lambda}^{\phi}$ will be an eigenvector of $\left(M^{\phi}\right)^{*}$ to eigenvalue $\lambda$. If $\lambda$ is an eigenvalue of $M^{\prime}$ and if $f_{\lambda}$ is an 
eigenvector for $M^{\prime}$ to eigenvalue $\lambda$, it must be that $f_{\lambda}=c k_{\lambda}^{\phi}$ for some $c \in \mathbb{C}$ since $\operatorname{Ker}\left(\left(M^{\phi}\right)^{*}-\lambda\right)=\mathbb{C}\left\{k_{\lambda}^{\phi}\right\}$ is one dimensional and $\left(M^{\phi}\right)^{*}$ extends $M^{\prime}$.

Given the Cayley transform $V^{\phi}=\mu\left(M^{\phi}\right)=\left(M^{\phi}-i\right)\left(M^{\phi}+i\right)$ of $M^{\phi}$, we have that $\operatorname{Dom}\left(V^{\phi}\right)=\operatorname{Ran}\left(M^{\phi}+i\right)$ and $\operatorname{Ran}\left(V_{\phi}\right)=\operatorname{Ran}\left(M^{\phi}-i\right)$. Let $\psi_{ \pm}$be fixed non-zero vectors in $\operatorname{Ran}\left(M^{\phi} \mp i\right)^{\perp}=\operatorname{Ker}\left(\left(M^{\phi}\right)^{*} \pm i\right)$ which have the same norm, $\left\|\psi_{+}\right\|=\left\|\psi_{-}\right\|$. Recall that the family of all unitary extensions of $V^{\phi}$ can be labeled by a single real parameter $\alpha \in[0,2 \pi$ ) as follows (see, for example [5,6], or [19]). Given any such an $\alpha$, define

$$
U^{\phi}(\alpha):=V^{\phi} \oplus e^{i \alpha}\left\langle\cdot, \psi_{+}\right\rangle_{\phi} \psi_{-} .
$$

As $\alpha$ ranges in $[0,2 \pi), U^{\phi}(\alpha)$ covers all possible unitary extensions of $V^{\phi}$, and the set of all $M^{\phi}(\alpha):=\mu^{-1}\left(U^{\phi}(\alpha)\right)$ for $\alpha \in[0,2 \pi)$ is the family of all self-adjoint extensions of $M^{\phi}$. In what follows, we choose $\psi_{-}=i 2 \pi k_{i}^{\phi}$ and $\psi_{+}=-C_{\phi} \psi_{-}$. Replacing $\psi_{ \pm}$by $c \psi_{ \pm}$where $c \neq 0$ does not change $U(\alpha)$.

The domain of $M^{\phi}(\alpha)$ can then be decomposed as:

$$
\operatorname{Dom}\left(M^{\phi}(\alpha)\right)=\operatorname{Dom}\left(M^{\phi}\right) \dot{+} \mathbb{C}\left\{\psi_{+}+e^{i \alpha} \psi_{-}\right\}
$$

[10, Section 80], where $\dot{+}$ denotes the non-orthogonal direct sum of linearly independent subspaces, and $\mathbb{C}\{\psi\}$ is the one dimensional linear span of a vector $\psi$. It follows that a point $x \in \mathbb{R} \backslash \sigma_{e}\left(M^{\phi}\right)$ will belong to $\sigma_{p}\left(M^{\phi}(\alpha)\right)$ if and only if there is a non-zero $c \in \mathbb{C}$ such that $f=-i 2 \pi k_{x}-c\left(\psi_{+}+e^{i \alpha} \psi_{-}\right)$belongs to $\operatorname{Dom}\left(M^{\phi}\right)$. If $f \in \operatorname{Dom}\left(M^{\phi}\right)$ then for any $z \in \mathbb{U}$ it follows that $\left\langle M^{\phi} f, k_{z}^{\phi}\right\rangle_{\phi}=z f(z)$. Alternatively,

$$
M^{\phi} f(z)=\left(M^{\phi}\right)^{*} f(z)=-i 2 \pi x k_{x}(z)-i c\left(\psi_{+}(z)-e^{i \alpha} \psi_{-}(z)\right) .
$$

Equating these two expression for $M^{\phi} f(z)$ yields

$$
\begin{aligned}
& z \frac{1-\overline{\phi(x)} \phi(z)}{z-x}-z c \frac{\phi(z)+\phi(i)}{z-i}-z c e^{i \alpha} \frac{1-\overline{\phi(i)} \phi(z)}{z+i} \\
& \quad=x \frac{1-\overline{\phi(x)} \phi(z)}{z-x}-i c \frac{\phi(z)-\phi(i)}{z-i}-i c e^{i \alpha} \frac{1-\overline{\phi(i)} \phi(z)}{z+i} .
\end{aligned}
$$

This expression can be simplified to yield

$$
0=\left(1+c \phi(i)+c e^{i \alpha}\right)-\phi(z)\left(\overline{\phi(x)}+c-c \overline{\phi(i)} e^{i \alpha}\right) .
$$

Since this must hold for all $z \in \mathbb{U}$, both of the bracketed terms must vanish separately, leading to the expression

$$
\phi(x)=\frac{e^{i \alpha}-\phi(i)}{1-\overline{\phi(i)} e^{i \alpha}} .
$$


Note that the right hand side of this formula has modulus 1 , as it must since the angular derivative of $\varphi$ at $\mu(x)$ exists.

\subsubsection{Summary of Results}

In summary, we have proven that $\sigma\left(M^{\phi}(\alpha)\right)=\sigma_{e}\left(M^{\phi}\right) \cup \sigma_{p}\left(M^{\phi}(\alpha)\right)$ where $\sigma_{e}\left(M^{\phi}\right)=\operatorname{supp}(\phi) \cap \mathbb{R}$ and $\sigma_{p}\left(M^{\phi}(\alpha)\right)=\left\{x \in \mathbb{R} \backslash \sigma_{e}\left(M^{\phi}\right) \mid \phi(x)=\frac{e^{i \alpha}-\phi(i)}{1-\overline{\phi(i)} e^{i \alpha}}\right\}$. In particular if $\phi(i)=0$ it follows that $\sigma_{p}\left(M^{\phi}(\alpha)\right)=\left\{x \in \mathbb{R} \backslash \sigma_{e}\left(M^{\phi}\right) \mid \phi(x)=e^{i \alpha}\right\}$. Here recall that $M^{\phi}(\alpha)$ is the inverse Cayley transform of $U^{\phi}(\alpha)$, as given in (4.2), with the specific choice of deficiency vectors $\psi_{-}=k_{i}^{\phi}, \psi_{+}=-C_{\phi} \psi_{-}$.

Remark 4.1.2 It is a simple calculation to verify that if $x, y \notin \sigma_{e}(M), x \neq y$, then $\left\langle k_{x}^{\phi}, k_{y}^{\phi}\right\rangle_{\phi}=0$ if and only if $\phi(x)=\phi(y)$. Indeed, this inner product is equal to $k_{x}^{\phi}(y)=\frac{1}{2 \pi} \frac{1-\overline{\phi(x)} \phi(y)}{y-x}$, and since $x, y \notin \sigma_{e}(M),|\phi(x)|=|\phi(y)|=1$, by Remark 4.0.7.

\section{5 de Branges Spaces}

Recall that a de Branges function $E$ is an entire function which obeys $|E(z)|>|E(\bar{z})|$ for all $z \in \mathbb{U}$. Given such a function the de Branges space $\mathcal{H}(E)$ is defined as the space of all entire functions $f$ such that $f / E$ and $f^{*} / E$ belong to $H^{2}(\mathbb{U})$ [23, Prop. 2.1]. The space $\mathcal{H}(E)$ is a Hilbert space with respect to the inner product $\langle f, g\rangle_{\mathcal{H}(E)}=$ $\langle f / E, g / E\rangle_{L^{2}(\mathbb{R})}[20]$.

Suppose that $\phi=F$ is an inner function which can be extended to a meromorphic function on $\mathbb{C}$. An inner function $F$ has this form if and only if its singular part has support only at the point at infinity, i.e. the singular part consists only an exponential term $e^{i \sigma z}, \sigma \geq 0$, and the zeroes of $F$ have no finite accumulation point on $\mathbb{R}$. Recall that an inner function $F$ has these properties if and only if there exists a de Branges function $E$ such that $F=\frac{E^{*}}{E}$ (Theorem 5.0.6). The following results characterizing de Branges spaces and de Branges functions can be found, for example, in [21,22].

Lemma 5.0.3 The map $U: \mathcal{H}(E) \rightarrow K_{E^{*} / E}^{2}$ defined by $U f=f / E$ is an isometry from $\mathcal{H}(E)$ onto $K_{E^{*} / E}^{2}$.

Remark 5.0.4 It is clear that if $E$ is a de Branges function, then $E^{*} / E$ is a meromorphic inner function. The defining inequality $|E(z)|>|E(\bar{z})|$ for $z \in \mathbb{U}$ ensures that the zeroes of $E$ are contained in the closure of the lower half plane and that $E^{*} / E$ is bounded by 1 in $\mathbb{U}$, and is unimodular on $\mathbb{R}$. Since $E$ is entire, its zeroes have no finite accumulation point, so that $E^{*} / E$ is analytic on some neighbourhood of each $x \in \mathbb{R}$, and meromorphic in $\mathbb{C}$.

If $w \in \mathbb{L}$ then $z-w$ is a de Branges function, also $e^{-i \sigma z}$ is a de Branges function for any $\sigma>0$. Any finite product of de Branges functions, or of a de Branges function with any entire function $G$ such that $G=G^{*}$, and $G$ has only real zeroes, is again a de Branges function. It follows easily that if $F(z)=e^{i \sigma z} \mathfrak{B}(z)$ is a meromorphic inner function where $\mathfrak{B}(z)=\prod_{n=1}^{N} \frac{z-z_{n}}{z-\bar{z}_{n}}$ is a finite Blaschke product, then 
$E(z)=\gamma G(z) e^{-i \sigma z / 2} \prod_{n=1}^{N}\left(z-\overline{z_{n}}\right)$, is a de Branges function satisfying $F=E^{*} / E$. Here, $\left\{z_{n}\right\} \subset \mathbb{U}, G$ is as described previously, and $\gamma \in \mathbb{T}$. The following theorems generalize these observations to the case where $\mathfrak{B}$ is an infinite product.

Theorem 5.0.5 (M. G. Krein) If $E$ is a de Branges function and $\left(\bar{z}_{n}\right)_{n \in \mathbb{N}}$ are its zeroes in $\mathbb{L}$ ordered so that $\left|z_{n}\right| \leq\left|z_{n+1}\right|$, then $\sum_{n=1}^{\infty}\left|\operatorname{Im}\left(\frac{1}{z_{n}}\right)\right|<\infty$ and,

$$
E(z)=\gamma G(z) e^{-i \sigma z} \prod_{n=1}^{\infty}\left(1-\frac{z}{\overline{z_{n}}}\right) e^{\frac{1}{2}\left(p_{n}(z)+p_{n}^{*}(z)\right)}
$$

where $\sigma>0,|\gamma|=1, p_{n}(z):=\sum_{k=1}^{n} \frac{1}{k \bar{z}_{n}^{k}} z^{k}$ and $G=G^{*}$ is an entire function whose zeroes lie on the real axis.

Theorem 5.0.6 If $F \in H^{\infty}(\mathbb{U})$ is inner, then $F=E^{*} / E$ for some de Branges function $E$ if and only if $F(z)=e^{i \sigma z} \mathfrak{B}(z) ; \sigma \geq 0$, where $\mathfrak{B}(z)$ is a Blaschke product whose zeroes have no finite accumulation point.

Let $F=e^{i \sigma^{\prime} z} \mathfrak{B}(z)$ be a meromorphic inner function with zeroes $\left\{z_{n}\right\}_{n=1}^{\infty}$, and $\sigma^{\prime} \geq 0$. A de Branges function $E$ satisfies $F=E^{*} / E$ if and only if both $\left\{\overline{z_{n}}\right\}_{n=1}^{\infty}$ is the set of non-real zeroes of $E$, and the constant $\sigma$ appearing in the canonical representation (5.1) of $E$ is equal to $\sigma^{\prime} / 2$. Hence, such an $E$ is determined uniquely by $F$ up to a unimodular constant $\gamma$ and an entire function $G$ which obeys $G=G^{*}$, and whose zeroes lie on $\mathbb{R}$.

These facts lead to the following representation theorem for regular simple symmetric operators with deficiency indices $(1,1)$.

Theorem 5.0.7 Let $B$ be a linear transformation with domain and range contained in a separable Hilbert space $\mathcal{H}$. Then $B$ is regular, closed and simple symmetric with deficiency deficiency indices $(1,1)$ if and only if it is unitarily equivalent to multiplication by $z$ in a de Branges space of entire functions. Equivalently, such a $B$ is unitarily equivalent to multiplication by $z$ in $K_{F}^{2}$ where $F \in H^{\infty}(\mathbb{U})$ is a meromorphic inner function.

By multiplication by $z$ in a de Branges space $\mathcal{H}(E)$, we mean the linear transformation in $\mathcal{H}(E)$ which acts as multiplication by $z$ on its domain, and which has no proper extension. The fact that multiplication by $z$ in any de Branges space $\mathcal{H}(E)$ is a closed regular simple symmetric linear transformation with deficiency indices $(1,1)$ is well known, see for example [6, Theorems 16-17], [20]. It remains to prove necessity.

Proof Suppose $B$ is closed, regular and simple symmetric with deficiency indices $(1,1)$. If $\omega_{B}$ is the characteristic function of $B$, then the regularity of $B$ and Theorem 4.0.5 imply that $\left|\omega_{B}(\lambda)\right|=1$, and that $\omega_{B}$ is analytic in a neighbourhood of $\lambda$ for any $\lambda \in \mathbb{R}$. It follows that $\omega_{B}$ is a meromorphic inner function and has the form $\omega_{B}(z)=e^{i \sigma z} \mathfrak{B}(z)$ where $\sigma \geq 0$, and $\mathfrak{B}(z)$ is a Blaschke product whose zeroes have no finite accumulation point. By Theorem 5.0.6, there is a de Branges function $E$ such that $\omega_{B}=E^{*} / E$. By Theorem 2.2.1, $B$ is unitarily equivalent to multiplication by $z$ 
in $K_{\omega_{B}}^{2}$, and since by Lemma 5.0.3, multiplication by $E$ is an isometry from $K_{\omega_{B}}^{2}$ onto the de Branges space $\mathcal{H}(E)$, it follows that $B$ is unitarily equivalent to multiplication by $z$ in $\mathcal{H}(E)$. The converse follows from the comment preceding this proof.

More precisely, by Theorem 2.2.1, the following is true.

Corollary 5.0.8 If $B$ is a regular simple symmetric linear transformation with deficiency indices $(1,1)$, then its characteristic function $\omega_{B}$ is a meromorphic inner function. For $\alpha \in \mathbb{D}$, let $\omega_{B}^{(\alpha)}:=\frac{\omega_{B}-\alpha}{1-\bar{\alpha} \omega_{B}}$. If $e^{i 2 \sigma(\alpha) z}$ is the singular part of $\omega_{B}^{(\alpha)}$ and $\left(z_{n}(\alpha)\right)_{n=1}^{\infty}$ are its zeroes, then $B$ is unitarily equivalent to multiplication by $z$ in any de Branges space $\mathcal{H}\left(E_{\alpha}\right)$ where

$$
E_{\alpha}(z)=\gamma(\alpha) G_{\alpha}(z) e^{-i \sigma(\alpha) z} \prod_{n=1}^{\infty}\left(1-\frac{z}{\overline{z_{n}(\alpha)}}\right) e^{\frac{1}{2}\left(p_{n}(\alpha ; z)+p_{n}^{*}(\alpha ; z)\right)},
$$

$\sigma(\alpha)>0,|\gamma(\alpha)|=1, p_{n}(\alpha ; z):=\sum_{k=1}^{n} \frac{1}{k \overline{z n n}_{n}(\alpha)} z^{k}$ and $G_{\alpha}$ is any entire function whose zeroes lie on the real axis and which obeys $G_{\alpha}=G_{\alpha}^{*}$.

The results of Sect. 3 applied to this particular case when $\phi=F=E^{*} / E$ for some de Branges function $E$ yields the following criterion for multiplication by $z$ to be a densely defined symmetric operator in $\mathcal{H}(E)$.

Theorem 5.0.9 Let $E$ be a de Branges function, and consider the representation of E given in Theorem 5.0.5. Then multiplication by $z$ in $\mathcal{H}(E)$ is densely defined if and only if at least one of the following two conditions holds: (i) $\sigma>0$, or (ii) $\sum_{n \in \mathbb{N}} \operatorname{Im}\left(z_{n}\right)=\infty$.

\subsection{The Spectra of Self-Adjoint Extensions of $M^{F}$}

Since the operator of multiplication, $M$, in $K_{F}^{2}$, where $F$ is a meromorphic inner function, has no essential spectrum, the spectra of any of its self-adjoint extensions is purely discrete. Recall that any symmetric linear transformation $B$ for which $B-z$ is bounded below for all $z \in \mathbb{C}$ is said to be regular. Since $M^{F}$, where $F$ is a meromorphic inner function is simple and $\sigma_{e}\left(M^{F}\right)=\{\infty\}$, it follows that $M^{F}$ is regular. Let the self-adjoint extension $M^{F}(\alpha)$ of $M^{F}$ be defined as in Sect. 4.1, i.e. choose the deficiency vectors $\psi_{-}=k_{i}^{F}$ and $\psi_{+}=-C_{F} \psi_{-}$. Since every extension has an infinite number of eigenvalues, it follows from Eq. (4.7) of Sect. 4.1 for the spectrum of $M^{F}(\alpha)$ that given any $\beta \in[0,2 \pi)$ there is an infinite number of points $x \in \mathbb{R}$ such that $F(x)=e^{i \beta}$.

Definition 5.1.1 For each $\beta \in[0,2 \pi)$, choose a point $x \in \mathbb{R}$ such that $F(x)=e^{i \beta}$ and let $M_{\beta}^{F}$ be that self-adjoint extension of $M^{F}$ for which $x \in \sigma\left(M_{\beta}^{F}\right)$.

The results of Sect. 4, show that $M_{\beta}^{F}$ is well defined, and that

$$
\sigma\left(M_{\beta}^{F}\right)=\left\{x \in \mathbb{R} \mid F(x)=e^{i \beta}\right\}
$$


Furthermore by inverting equation (4.7) of Sect. $4, M_{\beta}^{F}=M^{F}(\alpha)$ where $e^{i \alpha}=$ $\frac{F(i)+e^{i \beta}}{1+\overline{F(i)} e^{i \beta}}$. In particular, if $F(i)=0, M_{\beta}^{F}=M^{F}(\beta)$.

Since $F$ is unimodular on $\mathbb{R}$, we have $F(x)=e^{i \tau(x)}=E^{*}(x) / E(x)$ for a realvalued function $\tau$. It follows that the function $\tau: \mathbb{R} \rightarrow \mathbb{R}$ can be defined so that it is infinitely differentiable and has a local analytic extension about any point $x \in \mathbb{R}$. We will call such a function $\tau$ a phase function of $F$. Observe that the spectrum of $M_{\beta}^{F}$ can be written

$$
\sigma\left(M_{\beta}^{F}\right)=\{x \in \mathbb{R} \mid \tau(x)=\beta+2 \pi n ; n \in \mathbb{Z}\} .
$$

This implies in particular that any $x \in \mathbb{R}$ belongs to the spectrum of some self-adjoint extension of $M^{F}$. More precisely, the following results hold:

Theorem 5.1.2 ([5], [6, Theorem 2]) Let B be a closed symmetric operator densely defined in $\mathcal{H}$. If $B$ is simple, regular and has deficiency indices $(1,1)$, then the spectra of any one of its self-adjoint extensions consists of eigenvalues of multiplicity one with no finite accumulation point. Furthermore, the spectra of all of its self-adjoint extensions cover $\mathbb{R}$ exactly once.

Theorem 5.1.3 If $\tau: \mathbb{R} \rightarrow \mathbb{R}$ is such that $F(x)=e^{i \tau(x)}$, then $\tau^{\prime}(x)=2 \pi\left\|k_{x}^{F}\right\|^{2}>0$ and $\tau$ is a $C^{\infty}$ bijection of $\mathbb{R}$ onto $(-\infty, b)$, $(a, \infty)$ or $\mathbb{R}$, depending on whether the spectrum of each self-adjoint extension of $M^{F}$ is bounded above, bounded below or neither bounded above nor below, respectively. The phase function $\tau$ has a local analytic extension about any point $x \in \mathbb{R}$.

Note that if $E$ is a de Branges function such that $F=E^{*} / E$ then $\tau(x) / 2$ is a phase function associated with $E$, as defined in [20]. The fact that if one self-adjoint extension of $M^{F}$ is bounded above or below, then all are follows immediately from Krein's alternating eigenvalue theorem [8, pg. 19]:

Theorem 5.1.4 (Krein) Let B be a closed simple symmetric operator in $\mathcal{H}$ with deficiency indices $(1,1)$. Suppose that the interval $I \subset \mathbb{R}$ consists of regular points of $B$. Then, the eigenvalues of any two self-adjoint extensions $B^{\prime}$ and $B^{\prime \prime}$ of $B$ alternate in $I$.

Proof of Theorem 5.1.3 Since $F$ is analytic on a region containing $\mathbb{R}$, Theorem 3.1.1 implies that point evaluation at any point $x \in \mathbb{R}$ is a bounded linear functional in $K_{F}^{2}$; given any $f \in K_{F}^{2}$, and any $x \in \mathbb{R},\left\langle f, k_{x}^{F}\right\rangle=f(x)$, where $k_{x}^{F}(z)=\frac{i}{2 \pi} \frac{1-\overline{F(x)} F(z)}{z-x}$. It is straightforward to calculate that $0 \leq\left\|k_{x}^{F}\right\|^{2}=k_{x}^{F}(x)=\frac{1}{2 \pi i} \overline{F(x)} F^{\prime}(x)$, and since $e^{i \tau(x)}=F(x)$ it follows that $\tau^{\prime}(x)=-i \overline{F(x)} F^{\prime}(x)=2 \pi\left\|k_{x}^{F}\right\|^{2} \geq 0$. To show that $\tau$ is strictly increasing, and hence injective, it remains to show that $\left\|k_{x}^{F}\right\|>0$ is strictly positive for every $x \in \mathbb{R}$. To see that $k_{x}^{F} \neq 0$ for any $x \in \mathbb{R}$, recall that by definition, $k_{x}^{F}(z)=\frac{i}{2 \pi} \frac{1-\overline{F(x)} F(z)}{z-x}$ which is non-zero almost everywhere with respect to Lebesgue measure since $F$ is a non-constant inner function. Hence $\left\|k_{x}^{F}\right\|>0$, and $k_{x}^{F} \neq 0$ for any $x \in \mathbb{R}$. 
By (5.4) and the fact that $\tau$ is strictly increasing on $\mathbb{R}$, the spectra of each self-adjoint extension is bounded above or below if and only if the range of $\tau$ is bounded above or below. The phase function $\tau$ cannot be bounded both above and below as this, and the fact that the spectra of each self-adjoint extension of $B$ has no finite limit point (by Theorem 5.1.2) would imply that each self-adjoint extension has only a finite number of eigenvalues. Since each such self-adjoint extension is unbounded this is not possible. Now suppose that $\tau$ is bounded above and that $b=\sup _{x \in \mathbb{R}} \tau(x)=\lim _{x \rightarrow \infty} \tau(x)$. Then since $\tau$ is strictly increasing, $b$ is not in the range of $\tau$ and $\tau$ is onto $(-\infty, b)$. The other two cases are similarly easy to verify.

Remark 5.1.5 The spectrum of $M_{\beta}^{F}$ is $\sigma\left(M_{\beta}^{F}\right)=\{x \in \mathbb{R} \mid \tau(x)=\beta+2 \pi n$; $n \in \mathbb{Z} \cap \operatorname{Ran}(\tau)$ \}. Each $M^{F}$ is unitarily equivalent to $M^{\gamma F}$ where $\gamma$ is any unimodular constant. If $\tau_{\gamma}$ is a phase function of $\gamma F, \gamma F(x)=e^{i \tau_{\gamma}(x)}$, then there is always a $\gamma \in \mathbb{T}$ such that $\operatorname{Ran}\left(\tau_{\gamma}\right)=(-\infty, 0),(0, \infty)$ or $\mathbb{R}$, and $\operatorname{Ran}\left(\tau_{\gamma}\right) \cap \mathbb{Z}= \pm \mathbb{N}$ or $\mathbb{Z}$. For example, if $\operatorname{Ran}(\tau)=(a, \infty)$, let $\gamma=e^{-i a}$. Then $\tau_{\gamma}=\tau-a$ is a phase function for $\gamma F$ with range $(0, \infty)$.

Corollary 5.1.6 Given a phase function $\tau$ for $F$, let $\lambda: \mathbb{R} \rightarrow \mathbb{R}$ denote the monotonically strictly increasing $C^{\infty}$ function of $\operatorname{Ran}(\tau)$ onto $\mathbb{R}$ which is the inverse of $\tau$, $\lambda(\tau(x))=x$. Then $\sigma\left(M_{\beta}\right)=(\lambda(\beta+2 \pi n))_{n \in \mathbb{Z} \cap \operatorname{Ran}(\tau)}$.

Given an arbitrary densely defined simple symmetric operator $B$ with deficiency indices $(1,1)$, recall that, as in Sect. 4.1, all self-adjoint extensions $B(\alpha)$ of $B$ can be labeled by a single parameter $\alpha \in[0,2 \pi)$ where $B(\alpha):=\mu^{-1}(U(\alpha))$ is defined as the inverse Cayley transform of

$$
U(\alpha)=\mu(B) \oplus e^{i \alpha}\left\langle\cdot, \psi_{+}\right\rangle \psi_{-},
$$

and $\psi_{ \pm} \in \operatorname{Ker}\left(B^{*} \pm i\right)=\mathbb{C}\left\{\psi_{ \pm}\right\}$are chosen so that $\left\|\psi_{+}\right\|=\left\|\psi_{-}\right\| \neq 0$.

Corollary 5.1.7 Let $B$ be a regular simple symmetric linear operator with deficiency indices $(1,1)$ and characteristic function $\omega$. Let $\tau$ be a phase function for $\omega$. Then $\tau$ is a $C^{\infty}$ strictly monotonically increasing diffeomorphism of $\mathbb{R}$ onto its range, $\tau^{\prime}(x)>0$ for all $x \in \mathbb{R}$, and $\tau$ has a local analytic extension about any point $x \in \mathbb{R}$. If $B_{\beta}, \beta \in[0,2 \pi)$ is defined as that self-adjoint extension of $B$ such that there is an $x \in \sigma\left(B_{\beta}\right)$ so that $\omega(x)=e^{i \beta}$, then $\sigma\left(B_{\beta}\right)=\{\lambda(\beta+2 \pi n)\}_{n \in \mathbb{Z} \cap \operatorname{Ran}(\tau)}$, where $\lambda=\tau^{-1}$. If $B(\alpha)$ is defined as above, then there is a $\theta \in[0,2 \pi)$ such that $\beta=\alpha+\theta \bmod 2 \pi$, and $\psi_{ \pm}$can be chosen so that $\beta=\alpha$, i.e. so that $B_{\alpha}=B(\alpha)$.

The above corollary shows that the spectra of the self-adjoint extensions of $B$ behave very smoothly with respect to the parameter $\alpha$ labeling the self-adjoint extensions.

Proof The bulk of the corollary follows immediately from the previous results of this section, and the fact that $B$ is unitarily equivalent to $M^{\omega}$ in $K_{\omega}^{2}$. Assume, without loss of generality that $B=M^{\omega}$. We will verify the final assertion. By the results 4.1.1 of Sect. 4.1, and Definition 5.1.1, with the choice $\psi_{-}=k_{i}^{\phi}$ and $\psi_{+}=-C_{\phi} \psi_{-}$, $M_{\beta}^{\omega}=M^{\omega}(\beta)$ for all $\beta \in[0,2 \pi)$. If one makes a different choice $\psi_{ \pm}^{\prime}$ of deficiency 
vectors then $\psi_{ \pm}^{\prime}=c \chi_{ \pm} \psi_{ \pm}$, where $\psi_{ \pm}$are as before, $c=\frac{\left\|\psi_{ \pm}^{\prime}\right\|}{\left\|\psi_{ \pm}\right\|} \neq 0$ and $\chi_{ \pm} \in \mathbb{T}$. It follows that with this choice of deficiency vectors, if $\bar{\chi}_{+} \chi_{-}=e^{i \theta}$, then

$$
U^{\omega}(\alpha)=V \oplus e^{i \alpha}\left\langle\cdot, \chi_{+} \psi_{+}\right\rangle \chi_{-} \psi_{-}=V \oplus e^{\alpha+\theta}\left\langle\cdot, \psi_{+}\right\rangle \psi_{-}=U^{\omega}(\beta),
$$

where $U^{\omega}(\alpha)=\mu\left(M^{\omega}(\alpha)\right)$ and $\beta=\alpha+\theta \bmod 2 \pi$.

Remark 5.1.8 The function $\lambda=\tau^{-1}$ is the spectral function of the symmetric operator $B$, as defined and studied in Section 3 of [6]. Since the characteristic function $\omega_{B}$ of $B$ is only defined up to a unimodular constant, $\omega_{B}$ and the phase function $\tau$ can be chosen as described in Remark 5.1.5 so that $\operatorname{Ran}(\tau)=(-\infty, 0),(0, \infty)$ or $\mathbb{R}$. Furthermore, if the deficiency vectors of $B$ are chosen so that $B_{\alpha}=B(\alpha)$, then if $\lambda=\tau^{-1}, \sigma(B(\alpha))=\{\lambda(\alpha+2 \pi n) \mid n \in \mathbb{M}\}$ where $\mathbb{M}:=\mathbb{Z} \cap \operatorname{Ran}(\tau)= \pm \mathbb{N}$ or $\mathbb{Z}$.

\subsection{Subspaces with the Sampling Property}

Let $\mathcal{H}$ be a reproducing kernel Hilbert space of functions on $\mathbb{R}$, with point evaluation vectors $\delta_{x}, x \in \mathbb{R}$. We will call $\Lambda:=\left(\lambda_{n}\right)_{n \in \mathbb{M}}$ a total orthogonal sampling sequence for $\mathcal{H}$ if $\lambda_{n}<\lambda_{n+1}$ for all $n \in \mathbb{M}$ and $\left\{\delta_{\lambda_{n}}\right\}$ is a total orthogonal set, so that any $f \in \mathcal{H}$ can be reconstructed from its samples $\left\{f\left(\lambda_{n}\right)\right\}$ taken on the sampling sequence $\Lambda$. Here $\mathbb{M}= \pm \mathbb{N}$ or $\mathbb{Z}$. If $\mathcal{H}$ has a one-parameter family of total orthogonal sampling sequences $\Lambda(\alpha)$ which cover $\mathbb{R}$ exactly once, we will say that $\mathcal{H}$ has the $U(1)$ sampling property, and if $\mathcal{H}$ has the sampling property and there exists an orthogonal sampling sequence $\Lambda$ for $\mathcal{H}$ which has no finite accumulation points, we will say that $\mathcal{H}$ has the uniformly discrete sampling property. Reproducing kernel Hilbert spaces $\mathcal{H}$ of functions on $\mathbb{R}$ with the uniformly discrete sampling property seem to be of greater practical value for applications such as signal processing than those without this property. For example, suppose that one is given a RKHS $\mathcal{H}$ with the sampling property. One could then attempt to use $\mathcal{H}$ in the same way that the space of $\Omega$-bandlimited functions $B(\Omega)$ is used to sample and reconstruct continuous signals (see Sect. 1). Namely, given a continuous signal $f$, e.g. a music signal, approximate $f$ by an element $f_{\mathcal{H}}$ of $\mathcal{H}$. Since a music signal is a function of time, think of the real variable of elements of $\mathcal{H}$ as time. Using that $\mathcal{H}$ has a total orthogonal sampling sequence $\Lambda=\left(t_{n}\right)_{n \in \mathbb{M}}$, one can record the samples of $f_{\mathcal{H}}$ on $\Lambda$ to obtain the sampling sequence $\left(f_{\mathcal{H}}\left(t_{n}\right)\right)$, and then later reconstruct the approximation $f_{\mathcal{H}}$ to $f$ from this discrete sequence. If $\mathcal{H}$ does not have the uniformly discrete sampling property, the sequence of points $\left(t_{n}\right)$ has a finite accumulation point, and the above does not yield a practical method for sampling and reconstructing an approximation to the continuous signal $f$.

If $\phi$ is extreme, then as shown in Sect. 4.0.8, if $K_{\phi}^{2}$ has a total orthogonal sampling sequence then $\phi=F$ is an inner function. Moreover $\sigma_{e}(F) \backslash\{\infty\}=\varnothing$ if and only if $F$ is a meromorphic inner function. As observed at the beginning of Sect. 4.1, if $\lambda \notin \sigma_{e}(F)$ then point evaluation at $\lambda$ is a bounded linear functional in $K_{F}^{2}$. Hence if $F$ is meromorphic then $K_{F}^{2}$ is a reproducing kernel Hilbert space of functions on $\mathbb{R}$. Further suppose that $F$ is chosen so that $M^{F}$ is a densely defined symmetric operator (see Theorem 5.0.9 and use Theorem 5.0.6). In this case, if the self-adjoint extensions 
$M^{F}(\alpha)$ are defined as in Sect. 4.1, and $\sigma\left(M^{F}(\alpha)\right)=\left\{\lambda_{n}(\alpha)\right\}_{n \in \mathbb{M}}$, ordered so that $\lambda_{n}(\alpha)<\lambda_{n+1}(\alpha)$, then it follows from the results of the previous sections that for each $\alpha \in[0,2 \pi), \sigma\left(M^{F}(\alpha)\right)$ is a strictly monotonically increasing sequence with no finite accumulation point, the spectra of all the $M^{F}(\alpha)$ cover the real line exactly once, and for each $\alpha \in[0,2 \pi),\left\{k_{\lambda_{n}(\alpha)}^{F}\right\}_{n \in \mathbb{M}}$ is total orthogonal set of point evaluation vectors. Hence each subspace $K_{F}^{2}$ where $F$ is a meromorphic inner function for which $M^{F}$ is densely defined is a reproducing kernel Hilbert space with the $U(1)$ uniformly discrete sampling property. The fact that such $K_{F}^{2}$ have the $U(1)$ uniformly discrete sampling property is well known, and follows, for example from [20, Theorem 22] which uses different methods.

Remark 5.2.1 It is true that spaces $K_{G}^{2}$ for more general inner $G$ can also have total orthogonal sets of point evaluation vectors (see Sect. 4.0 .8 and [16]). However, if, for example, $\lambda \in \sigma_{e}\left(M^{G}\right)$ is an isolated point of the essential spectrum, then it is an accumulation point of the eigenvalues of every self-adjoint extension of $M^{G}$. In particular, $\lambda$ will be an accumulation point of any total orthogonal sampling sequence for $K_{G}^{2}$. Also if $\lambda \in \sigma_{e}(G)$ then point evaluation at $\lambda$ is a bounded linear functional in $K_{G}^{2}$ if and only if the angular derivative of $G \circ \mu^{-1}$ exists at $\mu(\lambda)$.

The following theorem applies the results of this section to provide a sufficient condition for a subspace $\mathcal{H} \subset L^{2}(\mathbb{R}, d v)$ to be a reproducing kernel subspace with the $U(1)$ uniformly discrete sampling property.

Theorem 5.2.2 Suppose that $v$ is a positive Borel measure which is absolutely continuous with respect to Lebesgue measure and let $M_{v}$ be the self-adjoint operator of multiplication by the independent variable in $L^{2}(\mathbb{R}, d v)$. Further assume that $\mathcal{H} \subset$ $L^{2}(\mathbb{R}, d v)$ is such that the Cayley transform $\mu\left(M_{\nu}\right)$ of $M_{v}$ is a unitary dilation of its compression to $\mathcal{H}$ and that $M_{v}$ has a regular simple symmetric restriction, $M_{v}^{\mathcal{H}}$, with deficiency indices $(1,1)$ to a linear subspace of $\mathcal{H}$. Then the following statements are true:

(1) $\mu\left(M_{\nu}\right)$ is the minimal unitary dilation of its compression to $\mathcal{H}$, and $v^{\prime}(x)>0$ almost everywhere.

(2) There is an isometric transformation $V$ which acts as multiplication by a measurable, locally $L^{1}$ function which takes $\mathcal{H}$ onto a de Branges space of entire functions. Hence the image of $M_{v}^{\mathcal{H}}$ under $V$ acts as multiplication by $z$.

(3) If $1 / v^{\prime}$ is a locally $L^{\infty}$ function and $M_{v}^{\mathcal{H}}$ is densely defined, then $\mathcal{H}$ itself is a reproducing kernel Hilbert space with the $U(1)$ uniformly discrete sampling property.

Note that in condition (1), the assumption that $v$ is absolutely continuous to Lebesgue measure, and that $v^{\prime}(x)>0$ almost everywhere with respect to Lebesgue measure, is equivalent to the assumption that $v$ is equivalent to Lebesgue measure. Recall that two measures are said to be equivalent if they have the same sets of measure zero.

This theorem is a strengthening of Theorem 14 of [6]. The proof of this theorem will make use of the following lemma. Given a semigroup $\mathfrak{S}$ of operators on a Hilbert space $\mathcal{H}$, recall that a subspace $S \subset \mathcal{H}$ is said to be semi-invariant for $\mathfrak{S}$ if $\left.\mathfrak{S}\right|_{S}$ is a semigroup. 
Lemma 5.2.3 (Sarason [24]) Let $\mathfrak{S}$ be a semi-group of operators on a Hilbert space $\mathcal{H}$. Then $S \subset \mathcal{H}$ is semi-invariant for $\mathfrak{S}$ if and only if $S=S_{1} \ominus S_{2}$ where $S_{2} \subset S_{1}$, and $S_{1}, S_{2}$ are invariant subspaces for $\mathfrak{S}$.

It is easy to verify that if $S_{2} \subset S_{1}$ are nested invariant subspaces for the semigroup $\mathfrak{S}$, then $S_{1} \ominus S_{2}$ is semi-invariant for $\mathfrak{S}$. Conversely, if $S$ is a semi-invariant subspace for $\mathfrak{S}$, then as in [24], one can show that $\overline{\mathfrak{S} S}=: S_{1}$ and $S_{1} \ominus S=: S_{2}$ are invariant subspaces of $\mathfrak{S}$ satisfying $S=S_{1} \ominus S_{2}$.

Before beginning the proof of Theorem 5.2.2, it will be useful to first recall some basic facts about unitary dilations of contractions, and to establish some notation.

\subsubsection{Unitary Dilations of Contractions and Semigroups of Contractions}

Let $T$ be a contraction on $\mathcal{H}$. Recall that a unitary operator $U$ on $\mathcal{K} \supset \mathcal{H}$ is called a unitary dilation of $T$ if $T^{k}=\left.P_{\mathcal{H}} U^{k}\right|_{\mathcal{H}}$ for all $k \in \mathbb{N} \cup\{0\}$. The dilation $U$ is called minimal if $\mathcal{K}$ is the closure of the linear span of $U^{k} \mathcal{H} ; k \in \mathbb{Z}$. Any contraction $T$ has a minimal unitary dilation, and this minimal unitary dilation is unique up to a certain natural unitary equivalence [26, Theorem 4.3].

If $1 \notin \sigma_{p}(T)$ where $\sigma_{p}(T)$ is the set of eigenvalues of $T$, then the Hardy functional calculus can be used to define $T(t):=\exp \left(i t \mu^{-1}(T)\right)$ for each $t \geq 0$. The functional calculus further implies $\mathfrak{S}:=\{T(t)\}_{t \geq 0}$ is a semi-group with respect to multiplication (in fact a representation of $([0, \infty),+$ ) since $T(t) T(s)=T(s+t) \forall s, t \geq 0$ and $T(0)=I)$, that $\|T(t)\| \leq 1$ for all $t \geq 0$, and that $t \mapsto T(t)$ is strongly continuous. Any semigroup of operators on $\mathcal{H}$ with these properties is called a strongly continuous one parameter contraction semigroup. Conversely, given any such one parameter contraction semigroup, $\mathfrak{S}=\{T(t)\}_{t \geq 0}$, the $\operatorname{limit} T:=\lim _{s \rightarrow 0^{+}} f_{s}(T(s))$ where $f_{s}(z):=\frac{z-1+s}{z-1-s}$ always exists in the strong operator topology. This limit, $T$, is a contraction on $\mathcal{H}$ such that $1 \notin \sigma_{p}(T)$, and such that $T(t):=\exp \left(i t \mu^{-1}(T)\right)$. The contraction $T$ is called the co-generator of $\mathfrak{S}$, and its inverse Cayley transform $\mu^{-1}(T)$ is called the generator. Analogously, a group $\mathfrak{g}=\{U(t)\}_{t \in \mathbb{R}}$ of operators on a Hilbert space $\mathcal{K}$ is called a strongly continuous one parameter unitary group if each $U(t)$ is unitary, if $t \mapsto U(t)$ is strongly continuous, and if $(\mathfrak{g}, \cdot)$ is a representation of $(\mathbb{R},+)$, i.e. $U(t) U(s)=U(t+s), U(0)=I$ and $U(-t)=U(t)^{-1}$ for all $s, t \in \mathbb{R}$. Such a strongly continuous unitary group of operators $\mathfrak{g}$ on $\mathcal{K} \supset \mathcal{H}$ is called a unitary dilation of a one parameter strongly continuous semigroup of contractions $\mathfrak{S}=\{T(t)\}_{t \geq 0}$ on $\mathcal{H}$ if $\left.P_{\mathcal{H}} U(t)\right|_{\mathcal{H}}=T(t)$ for all $t \geq 0$. Again, the dilation is called minimal if the linear span of $U(t) \mathcal{H} ; t \in \mathbb{R}$ is dense in $\mathcal{K}$, such a minimal dilation is unique up to a natural unitary equivalence, and every strongly continuous one parameter semigroup of contractions on $\mathcal{H}$ has a minimal unitary dilation [25, Chapter III, Sect. 8-9]. We refer the reader to $[25,26]$ for more on basic dilation theory.

By Stone's theorem, any strongly continuous one-parameter unitary group $\mathfrak{g}=\{U(t)\}_{t \in \mathbb{R}}$ of operators on $\mathcal{K}$ can be realized as $U(t)=e^{i t A}$ for some densely defined and closed self-adjoint operator $A$ acting in $\mathcal{K}$. The self-adjoint operator $A$ is said to generate the unitary group $\mathfrak{g}$. If $\mathfrak{S}_{\mathcal{K}}=\{U(t)\}_{t \geq 0}$, then this is clearly a strongly continuous semigroup, and its co-generator is $\mu(A)$. Moreover, the unitary group $\mathfrak{g}$ is a (minimal) unitary dilation of a strongly continuous one parameter contraction 
semigroup $\mathfrak{S}=\{T(t)\}_{s \geq 0}$ of operators on $\mathcal{H} \subset \mathcal{K}$ with co-generator $T$ if and only if $\mu(A)$ is a (minimal) unitary dilation of $T$. In general, $\mathfrak{g}$ is a unitary dilation of its compression $\mathfrak{S}_{S}:=\left\{\left.P_{S} U(t)\right|_{S}\right\}_{t \geq 0}$ to a subspace $S \subset \mathcal{K}$ if and only if $S$ is semi-invariant for $\mathfrak{S}_{\mathcal{K}}$. We will say $S$ is semi-invariant for $\mathfrak{g}$ if it is semi-invariant for the semi-group $\mathfrak{S}_{\mathcal{K}}$.

Let $M, M_{\nu}$, and $M_{E}$ denote the self-adjoint operators of multiplication by the independent variable in $L^{2}(\mathbb{R}), L^{2}(\mathbb{R}, d v)$ and $L^{2}\left(\mathbb{R},|E(x)|^{-2} d x\right)$, respectively. We will use the notation $\mathfrak{g}_{M}$, and $\mathfrak{g}_{v}$ and $\mathfrak{g}_{E}$ to denote the strongly continuous one-parameter unitary groups generated by $M, M_{v}$ and $M_{E}$.

By the Beurling-Lax theorem the invariant subspaces of the semigroup $\mathfrak{S}:=$ $\left\{\left.e^{i t M}\right|_{H^{2}(\mathbb{U})}\right\}_{t \geq 0}$ acting on $H^{2}(\mathbb{U})$ all have the form $F H^{2}(\mathbb{U})$, where $F$ is inner. It follows from Lemma 5.2.3 that each subspace $K_{F}^{2} \subset H^{2}(\mathbb{U})$ is semi-invariant for $\mathfrak{g}_{M}$. Moreover, it is clear that $\mathfrak{g}_{M}$ is the minimal unitary dilation of its compression $\mathfrak{S}_{M}$ to $K_{F}^{2}$. By Lemma 5.0.3, if $F=E^{*} / E$, where $E$ is a de Branges function, then multiplication by $E$ is an isometry of $K_{F}^{2}$ onto $\mathcal{H}(E)$. Clearly this isometry intertwines $e^{i t M}$ and $e^{i t M_{E}}$ for all $t \in \mathbb{R}$. It follows that $\mathfrak{g}_{E}$ is the minimal unitary dilation of its compression, $\mathfrak{S}_{E}:=\left\{\left.P_{\mathcal{H}(E)} e^{i t M_{E}}\right|_{\mathcal{H}(E)}\right\}_{t \geq 0}$, to $\mathcal{H}(E)$, and that $\mathfrak{S}_{E}$ is a semi-group. Here, $P_{\mathcal{H}(E)}$ is the projector of $L^{2}\left(\mathbb{R},|E(x)|^{-2} d x\right)$ onto $\mathcal{H}(E)$.

We now proceed with the proof of Theorem 5.2.2.

Proof of Theorem 5.2.2 The linear map $V$ of multiplication by $\sqrt{v^{\prime}(x)}$ is an isometry of $L^{2}(\mathbb{R}, d \nu)$ into $L^{2}(\mathbb{R})$ which maps $\mathcal{H}$ into a subspace $\mathcal{J} \subset L^{2}(\mathbb{R})$. If $M$ denotes the self-adjoint operator of multiplication by the independent variable in $L^{2}(\mathbb{R})$, then $V e^{i t M_{\nu}}=e^{i t M} V$ so that (by Remark 5.2.4 above) $\mathcal{J}$ is semi-invariant for the semigroup $\mathfrak{S}=\left\{e^{i t M}\right\}_{t \geq 0}$ acting on $L^{2}(\mathbb{R})$. By the Beurling-Lax theorem on invariant subspaces of this semi-group of operators on $L^{2}(\mathbb{R})$, and Lemma 5.2.3, it follows that $\mathcal{J}=S_{1} \ominus S_{2}$ where $S_{2} \subset S_{1}$ and each $S_{i}$ is either equal to $L^{2}(\Omega)$ where $\Omega$ is a Borel subset of $\mathbb{R}$ or $F H^{2}(\mathbb{U})$, where $F$ is a unimodular function. Let $M^{\mathcal{J}}$ denote the image of $M_{v}^{\mathcal{H}}$ under $V$.

The subspaces $S_{1}$ and $S_{2}$ cannot both have the form $L^{2}(\Omega)$, as then it would follow that $\mathcal{J}$ is itself invariant for $M$ so that $\mathcal{J}=L^{2}(\Lambda)$ where $\Lambda$ is a Borel subset of nonzero Lebesgue measure. It would follow that $M^{\prime}=\left.M\right|_{\mathcal{J}}$ is a self-adjoint extension of $M^{\mathcal{J}}$, and $\sigma\left(M^{\prime}\right)=\Lambda$, contradicting the fact that the spectrum of any self-adjoint extension of $M^{\mathcal{J}}$ is purely discrete. Furthermore, it cannot happen that only one of either $S_{1}$ or $S_{2}$ has the form $L^{2}(\Omega)$. First, if $\Omega$ is a proper non-trivial Borel subset of non-zero Lebesgue measure, then $L^{2}(\Omega)$ neither contains nor is contained in $F H^{2}(\mathbb{U})$ for any unimodular function $F$. Hence it cannot be that one of $S_{1}, S_{2}$ is equal to $L^{2}(\Omega)$ for such a set $\Omega$ while the other is equal to $F H^{2}(\mathbb{U})$. Suppose that either $S_{1}=L^{2}(\mathbb{R})$ and $S_{2}=F H^{2}(\mathbb{U})$ or $S_{1}=F H^{2}(\mathbb{U})$ and $S_{2}=\{0\}$. Then multiplication by $1 / F$ is an isometry from $\mathcal{J}=S_{1} \ominus S_{2}$ onto $S=H^{2}(\mathbb{L})$ or $=H^{2}(\mathbb{U})$, respectively, and the image $M^{S}$ of $M^{\mathcal{J}}$ under this isometry acts as multiplication by the independent variable. Consider the case where $S=H^{2}(\mathbb{U})$. The operator $\left.\mu(M)\right|_{H^{2}(\mathbb{U})}$ is an isometry on $H^{2}(\mathbb{U})$. Since 1 is not an eigenvalue of $\left.\mu(M)\right|_{H^{2}(\mathbb{U})}, M^{\prime}=\mu^{-1}\left(\left.\mu(M)\right|_{H^{2}(\mathbb{U})}\right)$ is a densely defined symmetric operator in $H^{2}(\mathbb{U})$. Furthermore, since $\mu\left(M^{\prime}\right)=$ $\left.\mu(M)\right|_{H^{2}(\mathbb{U})}$ is unitarily equivalent to the forward shift on $H^{2}(\mathbb{D})$, it is easy to see 
that $\operatorname{dim}\left(\operatorname{Dom}\left(\mu\left(M^{\prime}\right)\right)^{\perp}\right)=0$ and $\operatorname{dim}\left(\operatorname{Ran}\left(\mu\left(M^{\prime}\right)\right)^{\perp}\right)=1$ so that $\mu\left(M^{\prime}\right)$ and $M^{\prime}$ have deficiency indices $(0,1)$. Since $\mu\left(M^{S}\right)=\left.\mu(M)\right|_{\operatorname{Ran}\left(M^{S}+i\right) \subset H^{2}(\mathbb{U})}$ it follows that $\mu\left(M^{\prime}\right)$ is a closed isometric extension of $\mu\left(M^{S}\right)$. This yields a contradiction, since $\mu\left(M^{S}\right)$ has deficiency indices $(1,1)$ so that the only isometric extensions of $\mu\left(M^{S}\right)$ have the form $\mu\left(M^{S}\right) \oplus W$ on $S=\operatorname{Dom}\left(\mu\left(M^{S}\right)\right) \oplus \operatorname{Dom}\left(\mu\left(M^{S}\right)\right)^{\perp}$ where $W$ is a rank one isometry from the one-dimensional subspace $\operatorname{Dom}\left(\mu\left(M^{S}\right)\right)^{\perp}$ onto the one dimensional subspace $\operatorname{Ran}\left(\mu\left(M^{S}\right)\right)^{\perp}$. All such extensions have deficiency indices $(0,0)$ and are in fact unitary. A similar argument shows that $S$ cannot equal $H^{2}(\mathbb{L})$ either.

In conclusion $\mathcal{J}=G H^{2}(\mathbb{U}) \ominus F H^{2}(\mathbb{U})$ where $F, G$ are unimodular functions and $F H^{2}(\mathbb{U}) \subset G H^{2}(\mathbb{U})$. Since $F H^{2}(\mathbb{U}) \subset G H^{2}(\mathbb{U})$, it follows that for any $h \in H^{2}(\mathbb{U})$ there is a $h_{2} \in H^{2}(\mathbb{U})$ such that $F h=G h_{2}$. That is, given any $h \in H^{2}(\mathbb{U})$, $F / G h \in H^{2}$. This implies that $F / G$ is an inner function. Hence $\mathcal{J}=G\left(H^{2}(\mathbb{U}) \ominus\right.$ $\left.F / G H^{2}(\mathbb{U})\right)=G K_{F / G}^{2}$. Since elements of $\mathcal{J}$ have support on all of $\mathbb{R}$ it follows that $v^{\prime}(x)>0$ almost everywhere $x \in \mathbb{R}$. Furthermore, since $\mathfrak{g}_{M}$ is the minimal unitary dilation of its compression to $K_{F / G}^{2}$ and hence also of its compression to $\mathcal{J}$, it follows that $\mathfrak{g}_{v}$ is the minimal unitary dilation of its compression to $\mathcal{H}$.

Multiplication by $1 / G$ is an isometry of $\mathcal{J}$ onto the subspace $\mathcal{K}:=K_{F / G}^{2}=H^{2}(\mathbb{U}) \ominus$ $F / G H^{2}(\mathbb{U})$, and the image $M^{\mathcal{K}}$ of $M^{\mathcal{J}}$ under this isometry is again multiplication by the independent variable. Note by Remark 2.2.3, that $M^{\mathcal{K}}=M^{K_{F / G}^{2}}=M^{F / G}$, where $M^{F / G}$ is the symmetric linear transformation of multiplication by $z$ in $K_{F / G}^{2}$ as defined in previous sections. The transformation $M^{F / G}$ is simple with deficiency indices $(1,1)$. Since $M$ and hence $M^{\mathcal{K}}=M^{F / G}$ is regular, it follows as in the proof of Theorem 5.0.7 that $F / G$ is a meromorphic inner function, that there is a de Branges function $E$ such that $F / G=E^{*} / E$, and that multiplication by $E$ is an isometry of $K_{F / G}^{2}$ onto $\mathcal{H}(E)$. In summary, if $\widetilde{V}$ denotes the operator of multiplication by $v:=\sqrt{v^{\prime}} \frac{E}{G}$, then $\widetilde{V}$ is an isometry of $\mathcal{H}$ onto $\mathcal{H}(E)$ that takes $M^{\mathcal{H}}$ onto the symmetric operator of multiplication by $z$ in $\mathcal{H}(E)$.

To prove the third and final statement, let $\widetilde{v}$ be a member of the equivalence class of $v$ which is bounded below on any finite interval. Then if $k_{x}$ is the point evaluation vector at $x \in \mathbb{R}$ for $\mathcal{H}(E)$, it is easy to see that $\delta_{x}:=(\overline{\widetilde{v}(x)})^{-1} \widetilde{V}^{*} k_{x}$ is such that for any $f \in \mathcal{H},\left\langle f, \delta_{x}\right\rangle=f(x)$ almost everywhere. Identifying each $f$ in $\mathcal{H}$ with that member of its equivalence class for which this is true everywhere, we see that $\mathcal{H}$ is a reproducing kernel Hilbert space. The fact that $\mathcal{H}$ has the uniformly discrete $U(1)$ sampling property follows from the fact that any $\mathcal{H}(E)$ (or equivalently $K_{F}^{2}$ where $F=E^{*} / E$ ) in which multiplication by $z$ is densely defined has this property.

Remark 5.2.5 In the above theorem, the assumption that $M_{v}$ has a regular simple symmetric restriction with deficiency indices $(1,1)$ to a linear subspace of $\mathcal{H}$ can be replaced with the assumption that the essential spectrum of the compression of $\mu\left(M_{v}\right)$ to $\mathcal{H}$ consists of the singleton $\{1\}$. If $\sigma_{e}\left(\left.P_{\mathcal{H}} \mu\left(M_{\nu}\right)\right|_{\mathcal{H}}\right)$ contains only the point 1 , then $\mathcal{H}$ cannot be invariant for $M_{\nu}$, and hence is not reducing for $\mathfrak{g}_{v}=\left\{e^{i t M_{v}}\right\}_{t \in \mathbb{R}}$. It cannot be invariant or co-invariant for $\mathfrak{S}:=\left\{e^{i t M_{v}}\right\}_{t \geq 0}$ either, as this would imply 
that it is invariant or co-invariant for $\mu\left(M_{\nu}\right)$. It would follow that the compression of $\mu\left(M_{v}\right)$ to $\mathcal{H}$ is either an isometry or a co-isometry, and hence its spectrum would be the closed unit disc. In particular, the entire unit circle would belong to the essential spectrum of the compression of $\mu\left(M_{\nu}\right)$ to $\mathcal{H}$, contradicting our initial assumptions. As in the proof of the theorem above, this and the assumption that $\mathcal{H}$ is semi-invariant for $\mathfrak{g}_{v}$ then implies that there is a unitary $U$ from $\mathcal{H}$ onto a model subspace $K_{F}^{2}$, where $F$ is an inner function, such that $U$ acts as multiplication by a locally $L^{1}$ function. As has been demonstrated earlier, $M$ has a unique simple symmetric restriction $M^{F}$ with deficiency indices $(1,1)$ to a linear subspace of $K_{F}^{2}$, and hence $M_{v}$ has such a restriction $M_{v}^{\mathcal{H}}=U^{*} M^{F} U$ to a linear subspace of $\mathcal{H}$. The assumption that $\sigma_{e}\left(\left.P_{\mathcal{H}} \mu\left(M_{v}\right)\right|_{\mathcal{H}}\right)$ contains only the point 1 further implies that $M^{F}$ and hence $M_{v}^{\mathcal{H}}$ must be regular.

Theorem 5.2.6 Let $\mathcal{H}$ be a reproducing kernel Hilbert space of functions on $\mathbb{R}$ whose reproducing kernel is positive almost everywhere with respect to Lebesgue measure, $\left\|k_{x}\right\|^{2}>0$ a.e. $x \in \mathbb{R}$. Suppose that the operator of multiplication by the independent variable, $M^{\mathcal{H}}$ in $\mathcal{H}$ is a densely defined regular simple symmetric linear operator with deficiency indices $(1,1)$. Then there is an isometry $V$ which acts as multiplication by a function which is non-zero almost everywhere, which takes $\mathcal{H}$ onto a de Branges space $\mathcal{H}(E)$ of entire functions, and which takes $M^{\mathcal{H}}$ onto the symmetric operator of multiplication by $z$ in $\mathcal{H}(E)$.

The proof of this theorem relies on the theory of spectral representations of symmetric operators as developed by M. G. Krein (see for example [4,8] or [6, Section 2.2]), and tools developed in [6]. For the convenience of the reader, we provide a brief summary of the background theory needed in the proof of the above theorem.

Let $B$ be a closed regular simple symmetric linear operator with deficiency indices $(1,1)$ defined on a dense domain $\operatorname{Dom}(B) \subset \mathcal{H}$. Let $A$ be an arbitrary self-adjoint extension of $B$, and define the meromorphic vector-valued function $\psi_{z}:=(A-$ $i)(A-z)^{-1} \psi_{i}$ where $\psi_{i}$ is a fixed non-zero vector in $\operatorname{Ker}\left(B^{*}-i\right)$. Next define $\delta_{z}:=$ $\frac{\psi_{z}}{\left\langle\psi_{z}, \psi_{i}\right\rangle}$. By Lemma 2 of [6], $\left\langle\psi_{x}, \psi_{i}\right\rangle \neq 0$ for any $x \in \mathbb{R}$. Furthermore, $\delta_{z}$ is a meromorphic vector valued function with poles that lie off the real axis such that $\delta_{x} \in \operatorname{Ker}\left(B^{*}-x\right)$ for each $x \in \mathbb{R}$ (see for example Section 2.2 of [6]). One can then define a linear map $\Phi$ of $\mathcal{H}$ onto a certain vector space of meromorphic functions by $\Phi[f](z):=\left\langle f, \delta_{\bar{z}}\right\rangle$ for any $f \in \mathcal{H}$. It is easy to check that the image of $B$ under $\Phi$ acts as multiplication by the independent variable. Indeed, if $f \in \operatorname{Dom}(B)$, then $\Phi[B f](z)=\left\langle B f, \delta_{\bar{z}}\right\rangle=\left\langle f, B^{*} \delta_{\bar{z}}\right\rangle=z\left\langle f, \delta_{\bar{z}}\right\rangle=z \Phi[f](z)$.

By Corollary 5.1.7, and Remark 5.1.8, the deficiency vectors $\psi_{ \pm}$of $B$ can be chosen so that $\sigma(B(\alpha))=(\lambda(\alpha+2 \pi n))_{n \in \mathbb{M}}$ where $\lambda=\tau^{-1}$ is the spectral function of $B$, and is defined on $\operatorname{Ran}(\tau)=(-\infty, 0),(0, \infty)$ or $\mathbb{R}$ and $\mathbb{M}= \pm \mathbb{N}$ or $\mathbb{Z}$. Without loss of generality, assume that $\operatorname{Ran}(\tau)=\mathbb{R}$ so that $\mathbb{M}=\mathbb{Z}$. As in Section 4 of [6], one can endow the range of $\Phi$ with an inner product as follows.

Let $\rho$ be an arbitrary positive Borel probability measure on $[0,2 \pi]$, i.e. $\rho([0,2 \pi])=$ 1. Given any $f \in \mathcal{H}$, 


$$
\begin{aligned}
\langle f, f\rangle & =\int_{0}^{2 \pi}\langle f, f\rangle d \rho(\alpha) \\
& =\int_{0}^{2 \pi} \sum_{n \in \mathbb{Z}}\left\langle f, \delta_{\lambda(\alpha+2 \pi n)}\right\rangle\left\langle\delta_{\lambda(\alpha+2 \pi n)}, f\right\rangle \frac{1}{\| \delta_{\lambda(\alpha+2 \pi n) \|^{2}}} d \rho(\alpha) \\
& =\sum_{n \in \mathbb{Z}} \int_{0}^{2 \pi}\left\langle f, \delta_{\lambda(\alpha+2 \pi n)}\right\rangle\left\langle\delta_{\lambda(\alpha+2 \pi n)}, f\right\rangle \frac{1}{\left\|\delta_{\lambda(\alpha+2 \pi n)}\right\|^{2}} d \rho(\alpha) \\
& =\int_{-\infty}^{\infty}\left\langle f, \delta_{\lambda(x)}\right\rangle\left\langle\delta_{\lambda(x)}, f\right\rangle \frac{1}{\left\|\delta_{\lambda(x)}\right\|^{2}} d \rho(x) \\
& =\int_{-\infty}^{\infty}\left\langle f, \delta_{y}\right\rangle\left\langle\delta_{y}, f\right\rangle \frac{1}{\left\|\delta_{y}\right\|^{2}} d \rho(\tau(y)) \\
& =\int_{-\infty}^{\infty}|\Phi[f](y)|^{2} \frac{1}{\left\|\delta_{y}\right\|^{2}} d \rho(\tau(y)) .
\end{aligned}
$$

In the above, the measure $\rho$ is extended periodically to define a measure on $\mathbb{R}$. It follows that $\Phi$ can be viewed as an isometry of $\mathcal{H}$ onto a subspace of $L^{2}(\mathbb{R}, d \sigma)$ where $d \sigma(x)=d \rho(\tau(x))$.

Choose an arbitrary $z=r e^{i \beta} \in \mathbb{D}$, and define $d \rho_{z}(x)=P_{r}(\beta-x) d x$ where $P_{r}(\theta):=\frac{1-r^{2}}{1-2 r \cos \theta+r^{2}}$ is the Poisson kernel. Let $\sigma_{z}^{\prime}(x) d x:=d \sigma_{z}(x)=\frac{1}{\left\|\delta_{x}\right\|^{-2}}$ $d \rho_{z}(\tau(x))=\left\|\delta_{x}\right\|^{-2} P_{r}(\beta-\tau(x)) \tau^{\prime}(x) d x$. Let $M_{z}$ denote multiplication by the independent variable in $L^{2}\left(\mathbb{R}, d \sigma_{z}\right)$, and let $\Phi_{z}$ denote the map $\Phi$ viewed as an isometry from $\mathcal{H}$ onto $\mathcal{H}_{z}:=\Phi[\mathcal{H}] \subset L^{2}\left(\mathbb{R}, d \sigma_{z}\right)$. The following is taken from [6, Theorem 11].

Theorem 5.2.7 The subspace $\mathcal{H}_{z} \subset L^{2}\left(\mathbb{R}, d \sigma_{z}\right)$ is semi-invariantfor $\mathfrak{g}_{z}:=\left\{e^{i t M_{z}}\right\}_{t \in \mathbb{R}}$, and $\mathfrak{g}_{z}$ is the minimal unitary dilation of its compression to $\mathcal{H}_{z}$.

With the aid of the above facts, we are now ready to prove Theorem 5.2.6.

Proof For each $x \in \mathbb{R}$ such that $k_{x} \neq 0$, it is clear that $k_{x}$ is an eigenvector of $\left(M^{\mathcal{H}}\right)^{*}$ to eigenvalue $x$, where $M^{\mathcal{H}}$ denotes the symmetric operator of multiplication by the independent variable in $\mathcal{H}$. By the discussion preceding the proof, there is an isometry $\Phi$ of $\mathcal{H}$ onto a subspace $\mathcal{J} \subset L^{2}(\mathbb{R}, d \sigma)$ such that $d \sigma=\sigma^{\prime}(x) d x$, $\sigma^{\prime}, 1 / \sigma^{\prime}$ are locally $L^{\infty}$ functions, and such that $\mathcal{J}$ is semi-invariant for the semi-group $\mathfrak{S}:=\left\{e^{i t M_{\sigma}}\right\}_{t \geq 0}$ where $M_{\sigma}$ denotes multiplication by $x$ in $L^{2}(\mathbb{R}, d \sigma)$. Furthermore, the isometry $\Phi$ can be chosen such that if $f \in \mathcal{H}, \Phi[f](x)=\frac{\left\langle f, \psi_{x}\right\rangle}{\left\langle\psi_{i}, \psi_{x}\right\rangle}$ for $x \in \mathbb{R}$ where $\psi_{x} \in \operatorname{Ker}\left(\left(M^{\mathcal{H}}\right)^{*}-x\right)$. Since $\operatorname{Ker}\left(\left(M^{\mathcal{H}}\right)^{*}-x\right)=\mathbb{C}\left\{k_{x}\right\}$ for each $x \in \mathbb{R}$ such that $k_{x} \neq 0$, it follows that $\psi_{x}=c(x) k_{x}$ almost everywhere $x \in \mathbb{R}$ so that $\Phi[f](x)=\frac{f(x)}{\psi_{i}(x)}$ almost everywhere. 
In summary, $1 / \sigma^{\prime}$ is locally $L^{\infty}, \mathcal{J}$ is semi-invariant for $\mathfrak{S}=\left\{e^{i t M_{\sigma}}\right\}_{t \geq 0}$, and $M^{\mathcal{J}}:=\left.M_{\sigma}\right|_{\Phi \operatorname{Dom}\left(M_{\mathcal{H}}\right)}$ is regular simple symmetric and densely defined with indices $(1,1)$. By Remark 5.2.4 and Theorem 5.2.2, there is an isometry $V$ which acts as multiplication by a measurable function $v$ and which takes $\mathcal{J}$ onto a de Branges space $\mathcal{H}(E)$. In conclusion, multiplication of elements of $\mathcal{H}$ by $\frac{v(x)}{\psi_{i}(x)}$ is an isometry of $\mathcal{H}$ onto a de Branges space of entire functions, and which maps $M^{\mathcal{H}}$ onto multiplication by $z$ in $\mathcal{H}(E)$.

Corollary 5.2.8 In addition to the assumptions of the above theorem, suppose that $\mathcal{H} \subset L^{2}(\mathbb{R}, d v)$, where $v$ is a non-decreasing function of $x \in \mathbb{R}$. Let $E$ be any de Branges function such that $M^{\mathcal{H}}$, multiplication by $x$ in $\mathcal{H}$, is unitarily equivalent to $M^{E}$, multiplication by $z$ in $\mathcal{H}(E)$. Let $V$ be an isometry which takes $M^{F_{E}}$ onto $M^{\mathcal{H}}, F_{E}=E^{*} / E$, and acts as multiplication by the function $v(x)$. Then there exists $\phi \in B_{1}\left(H^{\infty}(\mathbb{U})\right)$ such that $\operatorname{Re}\left(\frac{1+F_{E} \phi}{1-F_{E} \phi}\right)$ is the Poisson integral of the measure $\mu$ where $d \mu(t)=|v(t)|^{2} d v(t)$.

This corollary uses the following fact [20, Problem 90, pg. 90]:

Lemma 5.2.9 Let $\mathcal{H}(E)$ be a de Branges space, and let $v: \mathbb{R} \rightarrow \mathbb{R}$ be a non-decreasing function such that

$$
\int_{-\infty}^{\infty}|f(x) / E(x)|^{2} d x=\int_{-\infty}^{\infty}\left|f(x) / E(x)^{2}\right| d v(x)
$$

for all $f \in \mathcal{H}(E)$. Then there exists a $\phi \in B_{1}\left(H^{\infty}(\mathbb{U})\right)$ such that

$$
\operatorname{Re}\left(\frac{1+F_{E}(z) \phi(z)}{1-F_{E}(z) \phi(z)}\right)=\frac{y}{\pi} \int_{-\infty}^{\infty} \frac{d \nu(t)}{(x-t)^{2}+y^{2}}
$$

where $z=x+i y \in \mathbb{U}$.

Proof of Corollary 5.2.8 Since $V$ is an isometry it follows that for any $f \in \mathcal{H}(E)$,

$$
\|f\|_{\mathcal{H}(E)}^{2}=\|f / E\|_{L^{2}(\mathbb{R})}^{2}=\int_{-\infty}^{\infty} \frac{|f(t)|^{2}}{|E(t)|^{2}}|v(t)|^{2} d v(t) .
$$

The corollary now follows from Lemma 5.2.9.

Open Access This article is distributed under the terms of the Creative Commons Attribution Noncommercial License which permits any noncommercial use, distribution, and reproduction in any medium, provided the original author(s) and source are credited. 


\section{References}

1. Kramer, H.P.: A generalized sampling theorem. J. Math. Phys. 38, 68-72 (1959)

2. Aldroubi, A., Gröchenig, K.: Beurling-Landau-type theorems for non-uniform sampling in shift invariant spline spaces. J. Four. Anal. Appl. 6, 93-103 (2000)

3. Everitt, W.N., Polkou, A.: Kramer analytic kernels and first-order boundary value problems. J. Comp. Appl. Math. 148, 29-47 (2002)

4. Silva, L.O., Toloza, J.H.: Applications of M. G. Krein's theory of regular symmetric operators to sampling theory. J. Phys. A 40, 9413-9426 (2007)

5. Kempf, A.: On fields with finite information density. Phys. Rev. D. 69, 124014 (2004)

6. Martin, R.T.W.: Symmetric operators and reproducing kernel Hilbert spaces. Op. Th. Comp. Anal., published online 17/04/2009

7. García, A.G., Hernándes-Medina, M.A.: Sampling theory associated with a symmetric operator with compact resolvent and de Branges spaces. Mediterr. J. Math. 2, 345-356 (2005)

8. Gorbachuk, M.L., Gorbachuk, V.I. (eds.): M.G. Krein's Lectures on Entire Operators. Birkhauser, Boston (1997)

9. Kaltenbäck, M., Woracek, H.: Pontryagin spaces of entire functions. Integr. Equ. Oper. Theory 33, 34-97 (1999)

10. Akhiezer, N.I., Glazman, I.M.: Theory of Linear Operators in Hilbert Space, Two Volumes Bound as One. Dover Publications, New York (1993)

11. Lifschitz, M.S.: A class of linear operators in Hilbert space. AMS Trans. 13, 61-83 (1960)

12. Reed, M., Simon, B.: Methods of Modern Mathematical Physics v.2: Fourier Analysis, Self-adjointness. Academic Press, Cambridge (1999)

13. Sarason, D.: Sub-Hardy Hilbert Spaces in the Unit Disk. Wiley, New York (1994)

14. Hoffman, K.: Banach Spaces of Analytic Functions. Prentice-Hall, Inc., Englewood Cliffs (1962)

15. Lotto, B.A., Sarason, D.: Multiplicative structure of de Branges's spaces. Rev. Math. Iber. 7(2), 183-220 (1991)

16. Fricain, E.: Bases of reproducing kernels in de Branges spaces. J. Funct. Anal. 226, 373-405 (2005)

17. Ahern, P.R., Clark, D.N.: On inner functions with $\mathrm{H}^{p}$ derivative. Mich. J. Math. 21, 115-127 (1974)

18. Lifschitz, M.S.: Isometric operators with equal deficiency indices. AMS Trans. 13, 85-103 (1960)

19. Martin, R.T.W., Kempf, A.: Quantum uncertainty and the spectra of symmetric operators. Ann. Appl. Math. 106, 349-358 (2009)

20. de Branges, L.: Hilbert Spaces of Entire Functions. Prentice-Hall, Englewood Cliffs (1968)

21. Baranov, A.D.: Bernstein's inequality in the de Branges spaces and embedding theorems. AMS Translations, pp. 21-49 (2003)

22. Havin, V., Mashreghi, J.: Admissable majorants for model subspaces of $\mathrm{H}^{2}$. Part I: Slow winding of the generating inner function. C. J. Math. 55, 1231-1263 (2003)

23. Remling, C.: Schrödinger operators and de Branges spaces. J. Funct. Anal. 196, 323-394 (2002)

24. Sarason, D.: On spectral sets having connnected complement. Acta Sci. Math. 26, 289-299 (1965)

25. Sz.-Nagy, B., Foiaş, C.: Harmonic analysis of operators on Hilbert space. American Elsevier publishing company, Inc., New York (1970)

26. Paulsen, V.: Completely Bounded Maps and Operator Algebras. Cambridge University Press, New York (2002) 